1 Characterizing and Contrasting the Microbial Ecology of Laboratory and Full-scale

\title{
Water Research
}

12

June 2016

${ }^{a}$ Department of Civil Engineering, University of Idaho, Moscow, ID, USA 83844-1022

${ }^{\mathrm{b}}$ Department of Statistics, University of Idaho, Moscow, ID, USA 83844-1104

* Correspondence concerning this paper should be addressed to him at Phone: (208) 885-7559; fax: (208) 885-6608; email: ecoats@uidaho.edu 


\section{Abstract}

16 The anthropogenic discharge of phosphorus (P) into surface waters can induce the

17 proliferation of cyanobacteria and algae, which can negatively impact water quality. Enhanced

18 biological P removal (EBPR) is an engineered process that can be employed to efficiently

19 remove significant quantities of $\mathrm{P}$ from wastewater. Within this engineered system, the mixed

20 microbial consortium (MMC) becomes enriched with polyphosphate accumulating organisms

21 (PAOs). To date much knowledge has been developed on PAOs, and the EBPR process is

22 generally well understood; nonetheless, the engineered process remains underutilized. In this

23 study, investigations were conducted using qPCR and Illumina MiSeq to assess the impacts of

24 wastewater (synthetic vs. real) on EBPR microbial ecology. While a strong relationship was

25 demonstrated between EBPR metrics (P:C; influent VFA:P) and excellent P removal across

26 diverse EBPR systems and MMCs, no such correlations existed with the specific MMCs.

27 Moreover, MMCs exhibited distinct clusters based on substrate, and qPCR results based on the

28 putative PAO Accumulibacter did not correlate with BLASTN eubacterial results for either

29 Accumulibacter or Rhodocyclaceae. More critically, PAO-based sequences aligned poorly with

30 Accumulibacter for both eubacterial and PAO primer sets, which strongly suggests that the

31 conventional PAO primers applied in FISH and qPCR analysis do not sufficiently target the

32 putative PAO Accumulibacter. In particular, negligible alignment was observed for PAO

33 amplicons obtained from a MMC performing excellent EBPR on crude glycerol (an atypical

34 substrate). A synthetic wastewater-based MMC exhibited the best observed BLASTN match of

35 the PAO amplicons, raising concerns about the potential relevance in using synthetic substrates

36 in the study of EBPR. 


\section{Introduction}

The anthropogenic discharge of excess nutrients (specifically nitrogen, N, and phosphorus,

P) into surface waters is being increasingly scrutinized for its adverse impact on water quality.

41 Among the concerns is the proliferation of cyanobacteria and algae. Commonly attributed to

42 excess P, Cyanobacteria outbreaks represent a serious health concern due to the production of

43 cyanotoxins (Brooks et al. 2016, Paerl 2014), which can be fatal to humans and animals if

44 ingested. Examples of Cyanobacteria blooms are numerous. In 2014, drinking water service was

45 cut to Oregon, Ohio (500,000+ customers) due to cyanotoxins in Lake Erie. More local to the

46 authors, similar issues occur annually in the Spokane River (Washington), a popular recreational

47 water body. In addition to the cyanotoxin concern, excess nutrients in aquatic environments ( $\mathrm{P}$ in

48 particular (Heathwaite and Sharpley 1999)) leads to advanced surface water body eutrophication,

49 which, in turn, can incur significant ecological and social damage (Pretty et al. 2003).

50 In an effort to reduce point-source $\mathrm{P}$ discharges, water resource recovery facilities

51 (WRRFs) are facing increasingly stringent permit limitations. Enhanced biological P removal

52 (EBPR) is an engineered WRRF process configuration that can be employed to achieve low

53 effluent $\mathrm{P}$ concentrations. Compared to chemical treatment alternatives, EBPR is more

54 environmentally sustainable (Coats et al. 2011c) and should be considered as a first line of

55 defense in achieving wastewater P removal. Theoretically, successful EBPR requires repeatedly

56 exposing a mixed microbial consortium (MMC) to an anaerobic environment in the presence of

57 volatile fatty acids (VFAs) followed by an aerobic environment. Within this engineered system,

58 the MMC becomes enriched with polyphosphate accumulating organisms (PAOs), which are the

59 putative organisms responsible for EBPR. PAOs uptake and store VFAs anaerobically as

60 polyhydroxyalkanoate (PHA); reducing equivalents for PHA synthesis are primarily provided by 
61 the catabolism of glycogen, while the ATP required for VFA uptake and activation is supplied by

62 glycogen catabolism and polyphosphate (polyP) hydrolysis (Lemos et al. 2003, Seviour et al.

63 2003). These anaerobic metabolisms result in an increase in bulk solution P. In the subsequent

64 aerobic environment, PAOs oxidize the stored PHA, which supplies the carbon and energy for

65 growth and the recovery of internal glycogen and polyP reserves. Through this cyclical process,

66 more $\mathrm{P}$ is stored than was released, resulting in the net removal of $\mathrm{P}$.

67 To advance EBPR process knowledge, research has sought to identify the microorganisms

68 constituting PAOs. Current knowledge on PAO microbiology is derived from the pioneering

69 work by Bond et al. $(1995,1999)$ and Hesselmann et al. (1999). Investigating the process at the

70 lab-scale and with synthetic wastewater, the former research team identified Betaproteobacteria

71 within the Rhodocyclus group (an unofficial taxonomic classification containing Rhodocyclus,

72 Azoarcus, and Zoogloea genera) enriched in EBPR systems. Subsequent investigations (Bond et

73 al. 1999), applying fluorescence in-situ hybridization (FISH) using 16S and 23S rDNA

74 oligonucleotide probes, confirmed the predominance of the Betaproteobacteria in EBPR systems

75 while also identifying other bacteria within the same class that were synthesizing PHA, but not

76 removing $\mathrm{P}$. It was concluded that the non-EBPR bacteria were glycogen accumulating

77 organisms (GAOs) that theoretically compete with PAOs in EBPR environments. Taking the

78 MMC characterization further, Hesselmann et al. (1999), through a combination of 16S rDNA

79 clone library analysis, polyP and PHA staining, and dot blot hybridization, identified a unique

80 bacterial species consistent with the PAO phenotype. While genetically similar to Rhodocyclus,

81 the identified bacterium did not exhibit the ability to grow phototrophically and was named

82 Candidatus Accumulibacter phosphatis (henceforth referred to as Accumulibacter); Crocetti et

83 al. (2000) drew similar conclusions. More recently, exploring the EBPR MMC based on inocula 
84 from Madison, WI (USA) and Queensland, Australia, Garcia Martin et al. (2006) published the

85 first metagenome of an Accumulibacter-enriched MMC, and affirmed that this organism is

86 related to Rhodocyclus (also noting it should be classified as its own genus).

87 Based on the work of Bond et al. (1995, 1999), Hesselmann et al. (1999), and Garcia

88 Martin et al. (2006), Accumulibacter is broadly recognized as the model PAO (Oehmen et al.

89 2007). Indeed, most EBPR microbial ecology research has focused on examining and

90 quantifying the relative abundance of Accumulibacter in EBPR MMCs, with a particular

91 emphasis on applying 16S rDNA-based FISH (e.g., (Albertsen et al. 2012, Nielsen et al. 2010,

92 Oehmen et al. 2007)) and, to a lesser degree, qPCR (e.g., (Coats et al. 2015, He et al. 2007, Mao

93 et al. 2015, Winkler et al. 2011)). These 16S rDNA-based investigations leveraged

94 oligonucleotide probes targeting Accumulibacter from Crocetti et al. (2000), wherein the

95 putative PAO-specific probes were developed based on DNA recovered from three synthetic

96 wastewater fed lab-scale EBPR MMC. However, the PAO phenotype has been observed in other

97 distinct taxonomic groups, including Tetrasphaera (Nguyen et al. 2011), Candidatus Halomonas

98 phosphatis (Nguyen et al. 2012) and Gemmatimonas aurantiaca (Zhang et al. 2003). Potentially

99 confounding the apparent uncertainty in affirming what microorganisms constitute PAOs are i)

100 the target-specific molecular methods (i.e., targeting Accumulibacter), which induces potential

101 bias, ii) the source of MMCs examined in microbial ecology studies, and iii) the associated

102 substrate on which the MMC were cultured. Most EBPR microbial ecology research has focused

103 at the laboratory scale and commonly with synthetic wastewater; such approaches deviate from

104 the full-scale environments to which this WRRF technology is applied. While next generation

105 sequencing methods (e.g., 454 pyrosequencing and Illumina MiSeq) have been applied in a

106 limited manner to more broadly characterize microbial communities in pilot or full-scale 
107 WRRFs, some of which were EBPR systems (Hu et al. 2012, Kim et al. 2013, Liu et al. 2016,

108 Wang et al. 2012, Wen et al. 2015), in all cases the focus was more on describing the MMC and

109 less on understanding the MMC within the context of EBPR.

110 While over the past 25 years substantial gains have been realized in understanding the

111 EBPR process at a macro- and micro-level, nonetheless the process remains underutilized at full-

112 scale largely due to concerns over process stability and reliability (Oehmen et al. 2007). From a

113 microbiological perspective, considering the relative diversity of putative PAOs observed in

114 EBPR studies (including, but not exclusively, Accumulibacter), the importance of any given

115 species or particular enrichment is in question; indeed, the collective body of EBPR

116 microbiological research suggests induced function of a diverse PAO enrichment is perhaps

117 more critical in realizing overall process success. However, in pursuit of an enhanced perspective

118 on MMC structure vs. induced function, there is a dearth of research contrasting important

119 parameters: operational scale and substrate (i.e., real vs. synthetic). Our research group has

120 extensively investigated the EBPR process (Al-Najjar et al. 2011, Coats et al. 2011a, Coats et al.

121 2011b, Coats et al. 2011c, Coats et al. 2015, Horgan et al. 2010, Winkler et al. 2011), with a

122 particular emphasis on expanding process knowledge and understanding through the

123 predominant use of real wastewater. Recent research demonstrated the successful use of crude

124 glycerol (an atypical EBPR substrate) in accomplishing excess P removal (Coats et al. 2015).

125 Moreover, investigations have collectively revealed variable PAO fractions in EBPR MMCs

126 (lab- and full-scale systems; applying qPCR based on Accumulibacter primers) seemingly

127 independent of substrate and process performance. Building upon these past investigations,

128 research was conducted to characterize EBPR performance and associated MMCs enriched

129 under differing substrate conditions and in lab- versus full-scale EBPR systems, with a particular 
130 emphasis on generating new insight on the structure-function relationship. In executing this 131 research, we posited:

132 (1) PAO research has largely centered on Accumulibacter, with methods for quantification 133 including FISH and qPCR using primers specific for Accumulibacter. However, the 16S rDNA 134 PAO results have not been interrogated beyond FISH and qPCR. Herein we consider Next Gen 135 Sequencing results related to EBPR MMC, applying both eubacterial and PAO primer sets; 136 results are evaluated based on phylotype classification.

137 (2) \% PAO in a MMC is not correlated with P removal (or any EBPR process metrics).

138 Research to date has yet to yield a convincing causal relationship, apart from the fact that PAOs 139 must be present. In other words, once a PAO population is are established, induced metabolisms 140 dictate process outcomes, and the \%PAOs themselves are not indicative of the success of the 141 process in general.

142 (3) Unique, potentially distinct communities are selected for lab- and full-scale systems.

143 Additionally, the MMC enriched by synthetic substrates are distinct from those enriched by real 144 wastewater.

\section{2. Materials and Methods}

146 2.1. Operating Conditions and System Assessments

147 Four laboratory-scale EBPR sequencing batch reactors (SBRs) and one full-scale WRRF system 148 (the Moscow, ID WRRF; an A²/O EBPR process) were evaluated. The SBRs were differentiated 149 by substrate: R-EBPR received only raw wastewater, V-EBPR received a traditional EBPR 150 substrate of raw wastewater and VFA-rich fermenter liquor, G-EBPR received raw wastewater

151 and crude glycerol, and S-EBPR received a synthetic substrate. Each SBR was operated with a 6 $152 \mathrm{hr}$ cycle consisting of the following periods: feed (5 min), anaerobic (1 hr), aerobic (4.25 hr), 
153 settle (30 min), and decant (10 min). A programmable logic controller was used to maintain SBR

154 operations. SBRs were operated at $2 \mathrm{~L}$ (maximum volume during the cycle) with effluent

155 decanted during each cycle and replaced with an equal volume of substrate to maintain an $18 \mathrm{hr}$

156 hydraulic residence time (HRT). The solids residence time (SRT) was controlled at $10 \mathrm{~d}$ by

157 automatically wasting $50 \mathrm{~mL}$ of mixed liquor per cycle at the end of each aerobic period prior to

158 settling. Air was introduced through stone diffusors to create aerobic conditions (dissolved

159 oxygen $(\mathrm{DO})>2 \mathrm{mg} \mathrm{L}^{-1}$ ) for the first $3.75 \mathrm{hr}$ of the aerobic period. The remainder of the aerobic

160 period proceeded without aeration to allow depletion of residual DO and ensure that anaerobic

161 conditions prevailed at the beginning of the subsequent cycle. Occasional DO checks throughout

162 the operational cycle were used to confirm each SBR conformed to target DO concentrations

163 (data not shown). Reactors were mixed with magnetic stir bars and operated at room temperature

164 without $\mathrm{pH}$ control. All pumping was performed using peristaltic pumps (Watson Marlow,

165 Wilmington, MA, USA). R-, V-, and G-EBPR have been the subjects in previous work (Coats et 166 al. 2015); however, the data presented herein is unique to this study.

167 The Moscow WRRF services a community of approximately 25,000 people with an

168 average influent flow rate of approximately $11,400 \mathrm{~m}^{3} \mathrm{~d}^{-1}$ (predominantly domestic wastewater).

169 The system consists of three anaerobic basins (each $787 \mathrm{~m}^{3}$ ), two pre-anoxic basins (each 1,363

$\left.170 \mathrm{~m}^{3}\right)$, an aerobic oxidation ditch $\left(6,814 \mathrm{~m}^{3}\right)$, and two $7,570 \mathrm{~m}^{3}$ secondary clarifiers, with influent

171 wastewater pre-treated through a $6 \mathrm{~mm}$ perforated plate screen followed by a vortex grit basin.

172 The WRRF operates at a total SRT of $15-18 \mathrm{~d}$. The return activated sludge flow rate is fixed at

$1734,088 \mathrm{~m}^{3} \mathrm{~d}^{-1}$, while the aerobic basin mixed liquor return flow rate to the first pre-anoxic basin is

174 controlled using a nitrate probe in the second pre-anoxic basin (target anoxic effluent $\mathrm{NO}_{3}-\mathrm{N}$ of

$\left.175 \quad 0.5-1.0 \mathrm{mg} \mathrm{L}^{-1}\right)$. 
The SBR and Moscow WRRF systems were each characterized on seven operational days

177 (with 5 October 2014 being operational day zero, the date on which S-EBPR was considered at

178 steady-state). System assessments took place on operational days 72, 86, 107, 121, 241, 263, and

179283 for the four SBRs and on operational days 225, 255, 270, 284, 304, 313, and 320 for the

180 Moscow WRRF.

181 2.2. Source of Microorganisms, Wastewater, and Crude Glycerol

182 SBR inocula were obtained from the Moscow WRRF. Each reactor was inoculated

183 independently; however, on operational day 147 S-and G-EBPR experienced a process upset due

184 to excess reactor wasting. G-EBPR was immediately re-inoculated from V-EBPR, while S-

185 EBPR was allowed to recover independently. Thereafter, SBR system assessments were

186 postponed for $93 \mathrm{~d}$ to reestablish steady-state.

187 Raw wastewater was obtained from the Moscow WRRF, downstream of screening and grit

188 removal. VFA-rich fermenter liquor was recovered from a bench-top fermenter fed thickened

189 primary solids from the Pullman, WA WRRF (Coats et al. 2011a). Crude glycerol (CG) was

190 acquired from the pilot-scale biodiesel production system operated by the Biological and

191 Agricultural Engineering Department at the University of Idaho (Moscow, ID, USA) (Thompson

192 and He 2006). CG was fed separately to the G-EBPR reactor, with a bulk solution CG

193 concentration $(\mathrm{t}=0)$ of approximately $1.0 \mathrm{Cmmol} \mathrm{L}^{-1}$. Fresh raw wastewater was obtained every

194 2-7 d, filtered through a $53 \mu \mathrm{m}$ stainless steel sieve (Gilson Company, Inc., Lewis Center, OH,

195 USA), and stored at $4^{\circ} \mathrm{C}$ until use. Bioreactor substrate tanks were replenished daily. The

196 unsterilized synthetic substrate for the S-EBPR reactor (based on Lu et al. (2006)) consisted of

197 (concentrations in the total feed volume prior to reactor addition): $\mathrm{NaCH}_{3} \mathrm{COOH}\left(281 \mathrm{mg} \mathrm{L}^{-1}\right)$;

$198 \mathrm{CH}_{3} \mathrm{CH}_{2} \mathrm{COOH}\left(173 \mathrm{mg} \mathrm{L}^{-1}\right) ; \mathrm{NH}_{4} \mathrm{Cl}\left(60.1 \mathrm{mg} \mathrm{L}^{-1}\right) ; \mathrm{CaCl}_{2}-\mathrm{H}_{2} \mathrm{O}\left(11.7 \mathrm{mg} \mathrm{L}^{-1}\right) ; \mathrm{K}_{2} \mathrm{HPO}_{4}(8.4 \mathrm{mg}$ 
$\left.199 \mathrm{~L}^{-1}\right) ; \mathrm{KH}_{2} \mathrm{PO}_{4}\left(8.4 \mathrm{mg} \mathrm{L}^{-1}\right) ; \mathrm{MgSO}_{4}-7 \mathrm{H}_{2}\left(52.8 \mathrm{mg} \mathrm{L}^{-1}\right)$; peptone $\left(2.2 \mathrm{mg} \mathrm{L}^{-1}\right)$; and yeast extract

200 (2.2 $\left.\mathrm{mg} \mathrm{L}^{-1}\right)$. Trace nutrients were added as per Lu et al. (2006). Nitrification was inhibited

201 through the combined addition of thiourea (15 mL (V-EBPR), $35 \mathrm{~mL}$ (G- and R-EBPR); stock 202 concentration $\left.40 \mathrm{~g} \mathrm{~L}^{-1}\right)$ and nitrapyrin $\left(\mathrm{ClC}_{5} \mathrm{H}_{3} \mathrm{NCCl}_{3} ; 1-2 \mathrm{~mL}(\mathrm{~V}-\mathrm{EBPR}), 1.5-2 \mathrm{~mL}(\mathrm{G}-\right.$ and R203 EBPR); stock concentration $0.5 \%$ in $95 \% \mathrm{EtOH}$ ) into the daily total substrate volume. Nitrapyrin 204 was added because thiourea proved to be only partially capable of inhibiting nitrification. Nitrate 205 was periodically measured to confirm nitrification was indeed inhibited.

\subsection{Analytical Techniques}

207 Samples were monitored for soluble reactive phosphate (SRP), volatile fatty acids (VFAs), 208 nitrate $\left(\mathrm{NO}_{3}\right)$, mixed-liquor suspended solids (MLSS), glycogen, PHA, and glycerol as described

209 in Coats et al. (2015). Measurement of pH was accomplished with a Thermo-Fisher Scientific

210 Accumet AP85 Waterproof pH/Conductivity Meter. DO measurements were collected using a

211 Hach HQ30d Meter with a LDO101 DO Probe.

\section{2.4. Quantitative Polymerase Chain Reaction (qPCR)}

213 qPCR was performed on genomic DNA recovered during each system assessment to

214 estimate the abundance of Accumulibacter (the model PAO) and glycogen accumulation 215 organisms (GAOs) relative to the total eubacterial population. Genomic DNA was extracted 216 using the MO BIO PowerSoil® DNA Isolation Kit (MO BIO Laboratories Inc., Carlsbad, CA 217 USA). Genomic DNA yield and purity was quantified using a Synergy H1 Multi-Mode Reader 218 (BioTek, Winooski, VT). Primer sets used to quantify total bacteria (Muyzer et al. 1993), 219 Accumulibacter (Crocetti et al. 2000, He et al. 2007), and GAOs (by targeting Candidatus 220 Competitbacter phosphatis, a model GAO) (Crocetti et al. 2002) are listed in Table S4. qPCR 221 settings were in accordance with Winkler et al. (2011). Triplicate qPCR was performed for all 
222 SBR samples both on a given 96 well plate (three replicate samples per plate) and on three plates

223 (thus 9 replicates per SBR sample). qPCR (triplicate on a 96 well plate; $\mathrm{n}=3$ ) was applied to

224 DNA extracted from the Moscow WRRF to align lab-scale operations with full-scale systems.

225 qPCR melting curves were evaluated to confirm a single melting peak, and agarose gel analysis

226 confirmed a single band for each primer set. Amplification efficiencies were calculated for each

227 primer set using baseline-corrected fluorescence data (StepOne software v2.0) and the

228 LinRegPCR program (Ramakers et al. 2003). Mean amplification efficiencies for the total

229 bacterial, PAO, and GAO primer sets were 95.6 $\pm 0.085 \%(\mathrm{n}=254), 89.7 \pm 0.088 \%(\mathrm{n}=250)$, and

$23082.3 \pm 0.074 \%(n=249)$ respectively. The cycle threshold was set at a constant value of 0.5 within

231 the log-linear region across all samples for determination of quantification cycle (Cq) values.

232 PAO and GAO relative abundances were estimated per Winkler et al. (2011).

233 2.5. DNA Sequence Analysis and Taxonomic Classification

234 Illumina sequencing was performed on genomic DNA recovered during each system

235 assessment. Bacterial 16S rRNA gene fragments were amplified and sequenced in accordance

236 with Hanson et al. (2016a). DNA amplicons were generated using two PCR rounds (round one

237 amplified the targeted region of the 16S rRNA gene and round two attached sequencing adapters

238 and sample barcodes) for eubacteria (Shen et al. 2016), PAOs (Crocetti et al. 2000, He et al.

239 2007), and GAOs (Crocetti et al. 2002); the primers are described in Table S6. The barcoded

240 amplicons were sequenced using an Illumina MiSeq instrument creating paired end 2x300 bp

241 libraries (Illumina, Inc., San Diego, CA).

242 Sequence analysis and taxonomic classification were performed following Hanson et al.

243 (2016a). Briefly, the Illumina MiSeq reads were demultiplexed and assigned to expected barcode

244 and primer sequences using the Python script dbcAmplicons 
245 (https://github.com/msettles/dbcAmplicons). After the primer sequences were trimmed, the reads

246 were joined into a single amplicon sequence using the application FLASH (Magoč and Salzberg

247 2011). The Ribosomal Database Project (RDP) Naïve Bayesian classifier was then used to assign

248 the joined sequences to phylotypes (Wang et al. 2007); assignment was made to the lowest

249 taxonomic rank with a bootstrap score $\geq 50 \%$. The relative abundance of individual phylotypes

250 in each sample was determined as the percentage of the corresponding sequence reads among the

251 total sequence reads in the sample.

252 As the RDP method is unable to identify Accumulibacter (Mao et al. 2015), an alternative

253 method was used to estimate its relative abundance. First, 27 16S rRNA gene sequences were

254 identified from He et al. (2007) as representatives of the 16S rRNA clades of Accumulibacter

255 (following Mao et al. (2015); summarized in Table S136). These reference sequences were then

256 classified with the RDP Bayesian classifier with a confidence threshold of $90 \%$. While

257 Accumulibacter has not been formally categorized, RDP classified all of the reference sequences

258 as belonging to the Rhodocyclaceae family (Table S136). Expecting the same classification for

259 Accumulibacter-related sequences in the samples, the subset of the joined sequences in each

260 sample classified as belonging to Rhodocyclaceae by RDP was identified using BLASTN (v.

261 2.3.0+) (Camacho et al. 2009) from a database consisting of the 27 Accumulibacter reference

262 sequences and the 16S rRNA gene sequences from the NCBI Nucleotides database assigned to

263 Rhodocyclaceae. Sequences were assigned to Accumulibacter if the top BLASTN result was one

264 of the 27 Accumulibacter reference sequences with at least $97 \%$ sequence similarity.

265 2.6. Data Analysis

266 Custom software (altvisngs; available at https://github.com/nguho/altvisngs) was used to 267 quantify the sample diversity and evenness indices, complete the rarefaction and hierarchical 
268 cluster analysis, and generate the taxon hierarchy with relative abundance, heatmap, and

269 summary bar plot images. Principal component analysis (PCA) was performed using R v3.2.5

270 (http://www.r-project.org/).

\section{$271 \quad 3 . \quad$ Results and Discussion}

272 3.1. Overall EBPR Performance

273 The influent and effluent P of each SBR were monitored regularly throughout the 274 operational period, with S-, V-, and G-EBPR achieving 95\% or greater P removal on average

275 (Table 1; Fig. S1). Consistent with our previous study (Coats et al. 2015), R-EBPR was less 276 effective in accomplishing $\mathrm{P}$ removal, achieving only 49\% $\mathrm{P}$ removal on average. Of the four

277 SBRs, G-EBPR most consistently achieved effluent $\mathrm{P}$ below $1 \mathrm{mg} \mathrm{P} \mathrm{L}{ }^{-1}$ over the operational 278 period (Fig. S1C). Even with several minor process upsets (Table 2), effluent P from S-EBPR 279 and V-EBPR was also less than $1 \mathrm{mg} \mathrm{P} \mathrm{L}^{-1}$ for most of the operational period (Fig. S1A and B).

280 While R-EBPR exhibited effluent P comparable to the other three SBRs at times, such

281 performance was ephemeral (Fig. S1D). Note that on operational days 268 and 269 the raw

282 wastewater substrate was contaminated by an unknown antibacterial agent; this wastewater

283 induced an upset in V-, G-, and R-EBPR, and was the only upset observed in G-EBPR over the 284 operational period (effluent $\mathrm{P}>3 \mathrm{mg} \mathrm{P} \mathrm{L}^{-1}$ ).

285 Regarding intra-cycle performance, as shown (Figs. S2-S5) the archetypal EBPR response 286 was exhibited by the MMCs in S- and V-EBPR (anaerobic P release with subsequent aerobic P 287 removal from bulk solution). In contrast, the MMCs in G- and R-EBPR did not consistently 288 cycle $\mathrm{P}$ in accordance with EBPR theory. The lack of anaerobic P release by the G-EBPR MMC, 289 coupled with its P removal in excess of 95\%, has been examined and discussed in detail 290 previously (Coats et al. 2015). All SBR MMCs consumed the available VFA substrate (and 
291 glycerol for G-EBPR) during the anaerobic period (data not shown, but consistent with the 292 previous study), and commensurately synthesized PHA (Table S1) in accordance with EBPR 293 theory. Also consistent with EBPR theory, glycogen was utilized anaerobically by all but R294 EBPR (Table S1).

295

296

297

298

299

300 311 the enrichment of PAOs over GAOs, although the ratios were lower than would be predicted by 312 the model of Filipe et al. (2001).

Two metrics are recognized as indicators of potential EBPR process success associated with a MMC enriched with PAOs: the anaerobic phosphorus released-to-VFA uptake (P:C) ratio (a characteristic of the PAO metabolic response; (Filipe et al. 2001, Smolders et al. 1994)), and the ratio of influent organic substrate to total P removed (Grady Jr. et al. 2011). For the P:C ratio, consistent anaerobic P release was observed in both S- and V-EBPR (Figs. S2, S3; Table 1), with average P:C ratios exceeding $0.19 \mathrm{Pmol} \mathrm{Cmol}^{-1}$ (total VFA basis). Conversely, G- and R-EBPR exhibited average $\mathrm{P}: \mathrm{C}$ ratios of 0.04 and $0.06 \mathrm{Pmol} \mathrm{Cmol}^{-1}$. The G-EBPR MMC did not always realize anaerobic P release (consistent with and as discussed in Coats et al. (2015)), nor did the R-EBPR MMC (Figs. S4, S5). Data from this and previous studies (Coats et al. 2011a, Coats et al. 2011b, Coats et al. 2015, Horgan et al. 2010, Winkler et al. 2011) were coupled with unpublished data obtained from our pilot-scale EBPR system (1:1000 scale of the Moscow WRRF) to examine the potential relationship of P:C with effluent P. As shown (Fig. 1), there appears to be a relationship between P:C and effluent P; excepting the S-EBPR data points, P:C ratios exceeding 0.2 resulted in effluent SRP less than $0.5 \mathrm{mg} \mathrm{L}^{-1}$. According to Lopez-Vazquez et al. (2007), and as summarized in Oehmen et al. (2007), such P:C ratios would appear to favor 
314 minimum amount of organic carbon (preferably VFAs) is necessary to realize the target PAO

315 enrichment capable of performing EBPR. The organic substrate is typically expressed on a

316 biochemical oxygen demand (BOD) or chemical oxygen demand (COD). Research indicates a

317 relationship between influent BOD and total $\mathrm{P}$ removed, with suggested minimum values

318 ranging from 15-20 BOD:P and 26-34 COD:P for high efficiency EBPR (summarized in (Grady

319 Jr. et al. 2011, WEF 2010)). While BOD and COD are measurements that can readily be tested at

320 full-scale WRRFs, both are gross aggregates of potential carbon substrate that do not specifically

321 identify the organic carbon substrate driving EBPR (VFAs). Coupling this study with previous

322 research (Coats et al. 2011a, Coats et al. 2011b, Horgan et al. 2010, Winkler et al. 2011) and

323 unpublished pilot- and full-scale operational data, results compiled in Fig. 2 indicate an influent

324 ratio of $15 \mathrm{mg}$ VFA (as COD) per mg $\mathrm{P}$ will both enrich for PAOs and induce the metabolic

325 responses necessary for EBPR. Both S-EBPR and V-EBPR exceeded the target ratio (Table 1;

326 Fig. 2). Conversely, R-EBPR exhibited inconsistent influent VFA:P ratios (VFAs were

327 commonly absent from the wastewater) and overall poor P removal. While the influent VFA:P

328 ratios for G-EBPR were similar to R-EBPR, performance was sustained through the addition of

329 crude glycerol (Coats et al. 2015). The VFA:P threshold suggested herein to achieve low effluent

330 P concentrations (based dominantly on real wastewater-based operations), being lower compared

331 with guidance in Grady et al. (2011) and the WEF Manual of Practice on Nutrient Removal

332 (WEF 2010), indicates that non-VFA substrates available in real wastewater also contributes to

333 EBPR process stability and resiliency. Indeed, non-VFA substrate (e.g., glucose; glycerol (Coats

334 et al. 2015)) can be used by microorganisms to directly generate energy (ATP) and PHA

335 anaerobically in support of EBPR; the coupled P:C ratios presented herein, being low as 
contrasted with theoretical models (Filipe et al. 2001, Smolders et al. 1994), further support this

337 observation (i.e., non-VFA substrate would not require polyP hydrolysis to generate energy for

338 uptake and catabolism, thereby reducing the P:C ratio).

339 3.3. $M M C$ Characterization with $q P C R$

340 qPCR was applied to assess the abundance of PAOs and GAOs relative to the total

341 eubacteria in the MMC (\%PAO and \%GAO, respectively) of each SBR and the Moscow WRRF

342 during each of the seven system assessments. Considering methodological repeatability, the

343 results were consistent within (triplicate) and across (triplicate) plates (i.e., low standard

344 deviation; Tables 3 and S3). The highest \%PAO values were observed in S-EBPR and Moscow

345 WRRF, with ranges of $0.61 \%$ to $15.14 \%$ and $3.97 \%$ to $17.54 \%$, respectively. Comparatively, V-,

346 G-, and R-EBPR exhibited markedly lower \%PAO. Indeed, on all but one date (operational day

347 283) V-EBPR sustained \%PAO less than 0.7\% (range of 0.12-2.6\%). Similarly, G-EBPR

348 exhibited \%PAO less than $0.82 \%$ (range of 0.26-0.82\%), while R-EBPR (which, again, exhibited

349 overall poor P removal) ranged from $0.11-1.14 \%$. Collectively, the observed \%PAO of the

350 MMCs were generally consistent with our previous investigations for the V-, G-, and R-EBPR

351 MMC (Coats et al. 2015), while lower than has been reported in other investigations (e.g., (He et

352 al. 2007, Winkler et al. 2011)). Perhaps more critically, there was no relationship between EBPR

353 performance and \%PAO.

354 Also consistent with Coats et al. (2015), the \%GAOs were generally low. The \%GAO in S-

355 EBPR was estimated at an order of magnitude lower than the \%PAO (Table 3). However, the

$356 \% \mathrm{GAO}$ in the V-EBPR MMC was quantitatively comparable to, and at times higher than, the

357 PAO fraction. GAOs were also present in the G-EBPR MMC, also approaching parity with

358 \%PAO. No GAOs were observed in the R-EBPR MMC (again consistent with Coats et al. 
359 (2015)). Comparatively, the \%GAO in the Moscow WRRF was substantially lower than the

$360 \%$ PAO (Table S3), and generally consistent with that observed in G-EBPR.

3.4. MMC characterization with Next Generation Sequencing

362

363

364

365

366

367

368

369

370

371

372

373

374

375

376

377

378

379

380

381

\subsubsection{Richness, Rarefaction, and Diversity Analyses}

Illumina sequencing targeting Eubacteria, PAOs, and GAOs was used to characterize the

MMCs in the SBRs and the Moscow WRRF. The total number of joined sequences obtained for the Eubacteria primer set are summarized in Table S7 (similar data for PAO and GAO primer sets is summarized in Tables S50 and S93); detailed phylotype data can be referenced via Table S5. The number of reads for the Eubacteria primer set ranged from 1,208 to 116,631; among the results there were two poor quality data sets (G-EBPR, day 283, 1,208 reads; V-EBPR, day 263, 6,428 reads). For Eubacteria, sequencing coverage depth was considered sufficient for each sample based on rarefaction analysis (Fig. S6, S7). However, coverage for the PAOs and GAOs was insufficient for most, but not all, samples (Figs. S76, S77, S136, S137). In executing the experimental design for the MiSeq analysis, genomic DNA pooling for Eubacterial, PAO, and GAO analyses was based on prior qPCR results (Coats et al. 2015, Winkler et al. 2011).

Ultimately, however, the analysis under-estimated the quantity of DNA needed to achieve full coverage for the PAO and GAO primer sets.

The Shannon index was calculated for the respective MMC to assess complexity. Three of the SBRs (V-, G-, and R-EBPR) exhibited similar complexity (average Shannon index of 3.903.94; range of 3.53-4.33; Table S7), while S-EBPR exhibited a slightly less complex microbial population (average Shannon index of 3.62; range of 3.36-3.9). In contrast, the Moscow WRRF MMC was the most complex (average Shannon index of 4.71). The Shannon index was selected for its value as a general complexity measure considering both richness and evenness (Hill et al. 

2003). On the Shannon evenness scale, all SBR averages were similar while the Moscow WRRF 383 again exhibited more complexity.

\subsubsection{Eubacteria Primer Set}

A comprehensive summary of the eubacterial-based MMC composition from the domain to genus level for each system assessment is presented in Figs. S14-S53 (see also Table S5). Table 4 summarizes the dominant phyla (averages across all operational days); the average microbial community composition in each SBR and the Moscow WRRF over the seven system assessments is presented in Figs. S21, S29, S37, S45, and S53. In all systems, Proteobacteria dominated the MMCs at the phylum level, followed by Bacteroidetes; no other phyla were, on average, observed at $>10 \%$. In contrast, nine phyla predominated within the minor category (1$10 \%)$, and substantive differences were observed across the MMC. Of the nine phyla, only Verrucomicrobia, Firmicutes, and Chloroflexi were common to all MMCs; unique to the Moscow WRRF was Nitrospirae, a nitrifying phyla that would not have been expected in the SBRs due to the imposed nitrification inhibition. The Moscow WRRF MMC exhibited the largest number of minor phyla (9), while S-EBPR exhibited the fewest (4). Similar phylum-level analysis of the respective MMCs for the PAOs (Table 4; Figs. S84-S123; Table S5) again revealed that Proteobacteria dominated across the tested reactors, although Actinobacteria and an unknown Bacteria were observed at $>10 \%$ in V-and G-EBPR; the PAO primer set targeted the Proteobacteria phyla. No minor phyla were observed in S-EBPR for the PAO primer set, while only 1-2 phyla were observed in the other four EBPR systems. For the GAO primer set, Proteobacteria dominated (except for R-EBPR, where an unknown Bacteria also predominated). At the class level for the eubacterial primers, Alphaproteobacteria and Betaproteobacteria were the only two observed in the S-EBPR MMC at >10\% (see Table S5; Figs. S14-S21). The 
V-, G-, and R-EBPR MMC, along with the Moscow WRRF, exhibited a similar composition,

406 although Betaproteobacteria dominated over Alphaproteobacteria; Sphingobacteria was also

407 observed at approximately $10 \%$, but $<4 \%$ in S-EBPR. Interrogating the MMCs at a lower rank

408 (order; Table 5), the Betaproteobacterium Rhodocyclales and the Alphaproteobacterium

409 Rhodobacterales were observed in S-EBPR at $>10 \%$; Flavobacteriales, from the Bacteroidetes

410 phylum, was observed at nearly $10 \%$ of the total population as well. In contrast,

411 Hydrogenophilales, from the Betaproteobacteria phylum, was more prominent in V-and R-

412 EBPR, while Sphingobacteriales (of the Bacteroidetes phylum) was dominant in R-EBPR and

413 also was the lone dominant order in the Moscow WRRF MMC. No dominant orders (>10\%)

414 were observed on average in G-EBPR across all operational days, although Sphingobacteriales

415 averaged $9.87 \%$. Note that the threshold for "minor" at the level of order was established at 5\%, 416 given the increased number of bacteria observed at $>1 \%$.

417 Finally, examining the MMC at the genus level, with the increased number of bacteria that 418 can be identified, the threshold for "dominance" was adjusted to 3\%. The S-EBPR MMC was 419 predominated by (in order, highest to lowest) Thauera, Flavobacterium, Ohtaekwangia, and

420 Propionivibrio. The V-EBPR MMC was predominated by Thiobacillus, Haliscomenobacter,

421 Thauera, Zoogloea, and Meganema at the genus level, while G-EBPR was predominated by

422 Saccharibacteria_genera_inc._sed., Haliscomenobacter, Methylobacillus, Zoogloea, and

423 Meganema. The R-EBPR MMC was enriched with three genera at $>3 \%$ of the population

424 (Thiobacillus, Thauera, and Meganema), while the Moscow WRRF only revealed one known 425 genus at $>3 \%$ (Haliscomenobacter).

426 The MiSeq results were also interrogated using multi-variate methods, which revealed

427 interesting contrasts. First considering a Bray-Curtis similarity analysis, at the genus level all 
428 samples clustered by reactor (Fig. S13), with the exception of V-. G-, and R-EBPR (day 283) and

429 V-EBPR (day 263). Both V-EBPR (day 263) and G-EBPR (day 283) exhibited low total reads

430 (Table S7), which likely explains the associated result, and V-EBPR (day 263) exhibited low

431 total reads relative to the other five sampled operational days. Considering the clustered results,

432 the V-and G-EBPR MMC exhibited the closest similarity, followed by R-EBPR, the Moscow

433 WRRF, and lastly S-EBPR (i.e., synthetic wastewater-based MMC was distinctly different from

434 all the real wastewater systems). Principal component analysis (PCA) confirmed these

435 observations (Fig. 3; Figs. S66-S75). As shown (Fig. 3), the MMC at the genus level exhibited

436 two distinct clusters within the first two PCA dimensions, with V-, G-, and R-EBPR uniquely

437 separated from that of S-EBPR in both dimensions. Moreover, the $95 \%$ confidence region

438 revealed much more variability within the real wastewater based MMC vs. S-EBPR. Combined,

439 the first two dimensions explained $21.61 \%$ of the variation among the sample sets. When the

440 Moscow WRRF samples were integrated into the analysis (Figs. S66-S75), while the

441 comparative distinction at the genus level between V-, G-, and R-EBPR vs. S-EBPR remained,

442 the relative separation becomes less pronounced while the Moscow MMC observations are quite

443 uniquely separate (Fig. S74). For this second PCA, the first two dimensions explained $22.63 \%$ of

444 the variation among the sample sets. In particular, the first principal component explains almost

$44515 \%$ of the variation.

\section{$446 \quad 3.4 .3 . \quad P A O$ and GAO Primer Sets}

447 Considering PAOs, while MiSeq coverage of the MMCs for most operational days was 448 considered inadequate (Figs. S76, S77), results on operational day 283 exhibited good coverage 449 for S- and V-EBPR; similarly, for G-EBPR the coverage was sufficient on operational days 121, 450241 , and 283. For these operational days, all MMC were nearly $100 \%$ dominated by 
451 Betaproteobacteria at the class level, which was an unsurprising result given that the model PAO 452 on which the primers are based is a Betaproteobacteria (He et al. 2007). At the genus level, the 453 S-EBPR MMC was dominated by Propionivibrio and Azospira (the former at $>80 \%$ ); both are of 454 the Rhodocyclaceae family. The V-EBPR MMC was similarly dominated by Propionivibrio at 455 the genus level on operational day 283 (>80\%), but Azospira was only $0.0034 \%$ of the MMC. 456 The G-EBPR MMC was also enriched with Propionivibrio (especially on day 121 at $83.7 \%$, 457 which also corresponded with the best rarefaction curve of all PAO data sets). While most other 458 data sets did not exhibit the coverage to justify significant conclusions, Propionivibrio 459 nonetheless was commonly observed (in many instances dominating; Tables S92). Functionally, 460 Propionivibrio is a fermentative bacterium with the capability to produce short-chain VFAs (i.e., 461 the optimal EBPR substrate) from polysaccharides (Zhou et al. 2015); given these metabolic 462 capabilities, certain species of Propionivibrio could be PAOs, although recent research has 463 identified one potential species (Candidatus Propionivibrio aalborgensis) as a putative GAO 464 (Albertsen et al. 2016). Other genera including Azonexus and Dechloromonas (within the 465 Rhodocyclaceae family of the Betaproteobacteria class) were observed, as was Bdellovibrio 466 (which is within the Bdellovibrionaceae family of the Deltaproteobacteria class). The Moscow 467 WRRF MMC was dominated by an unknown Rhodocyclaceae at the genus level, along with a 468 large fraction of Propionivibrio. Conversely, the R-EBPR MMC was dominated by 469 Dechloromonas.

470 Similar to the PAO MiSeq results, data obtained using the GAO primers did not exhibit 471 sufficient coverage across all samples (Fig. S136, S137). However, for V-EBPR on operational 472 day 241 and for G-EBPR on days 241, 263, and 283 the coverage was sufficient to draw

473 interpretations; additionally, the S-EBPR MMC on operational day 263 appeared to exhibit good 
474 overall coverage. The MMC were dominated by Gammaproteobacteria. Extrapolating to the

475 other GAO data sets, all MMC except R-EBPR consistently showed Gammaproteobacteria at $476>86.2 \%$.

477

478

479

480

481

482

483

484

485

486

487

488

489

490

491

492

493

494 relative abundance (Eubacterial primers) for both Accumulibacter and Rhodocyclaceae and the

495 qPCR data; moreover, in nearly all cases the qPCR \%PAOs exceeded MiSeq estimates based on

496 the Accumulibacter BLASTN data. Considering that all evaluated systems (with the exception of

\subsection{Estimating Accumulibacter and PAO Relative Abundance with Next Generation Sequencing}

MiSeq results were binned based on alignment, or lack thereof, with the 27 Accumulibacter sequences (He et al. 2007). To the authors' best knowledge, similar such studies on EBPR systems are few (two using ppk and qPCR (He et al. 2007, Mao et al. 2015); one using 454 pyrosequencing (Kim et al. 2013); one using qFISH (Albertsen et al. 2012)) and no such interrogation has been performed on MMCs in lab- or full-scale systems and across diverse wastewater substrates based on next generation sequencing data. For the Eubacterial data across all MMC, fractional Accumulibacter ranged from $0.0 \%$ to $21.45 \%$ (Table S137); while the range appears large, the second highest fraction was $4.03 \%$. Of significance, on many operational days the data suggests no Accumulibacter were present in the MMC; in particular, the G-EBPR showed no Accumulibacter, and R-EBPR was essentially zero as well. Targeting the family Rhodocyclaceae, the relative abundance ranged from $0.27 \%$ to $30.38 \%$; in contrast to the Accumulibacter results, MMC in G- and R-EBPR showed fractions comparable to that observed in V-EBPR, and higher than those observed in the Moscow WRRF. Oehmen et al. (2010) suggested that Accumulibacter fractions in real WRRFs would range from 5-20\%; results herein suggest this range is correct for Rhodocyclaceae but not Accumulibacter specifically.

Comparing the qPCR and BLASTN results, no correlation was observed between the 
R-EBPR) performed quality P removal (Table 2), it would appear that other non-Accumulibacter

498 species (including within the Rhodocyclaceae family) are PAOs, and these non-Accumulibacter

499 species are captured (amplified) by the model PAO primers and quantified via qPCR. To the best

500 of our knowledge, this is only the second study that interrogated real wastewater EBPR systems

501 for PAOs using both qPCR and next generation sequencing (454 pyrosequencing or Illumina

502 MiSeq); results of this study aligned with that of Mao et al. (2015), who similarly observed no

503 correlation between PAOs (applying 16S rDNA and primers for $p p k$ ) and 454 pyrosequencing

504 data.

\subsection{Specificity of the PAO Primer Set}

506 The BLASTN analysis was also carried out on the PAO primer set results to assess the 507 specificity of the PAO primer set for both Rhodocyclaceae and Accumulibacter (summarized in

508 Table S138). Unexpectedly, assignment of the sequences by RDP to Rhodocyclaceae was

509 variable. Five of the seven operational days for S-EBPR had more than $96 \%$ of the sequences

510 assigned to Rhodocyclaceae while none of the R-EBPR samples exceeded 88\%. Alignment of

511 the sequences with the 27 Accumulibacter sequences (He et al. 2007) was also variable. For

512 example, S-EBPR on operational day 283 had $98.17 \%$ of the Rhodocyclaceae assigned

513 sequences align with Accumulibacter, whereas G-EBPR was less than $1 \%$ on four of the seven

514 operational days (and did not exceed 11\%). As with the eubacterial primers, few to no sequences

515 from G- and R-EBPR aligned with Accumulibacter. These results further support that the model 516 PAO primers ultimately amplify non-Accumulibacter species (that may also be PAOs).

\section{3.7. Identifying other Potential PAOs}

518 With the unexpected P removal performance of G-EBPR (data presented herein and in

519 Coats et al. (2015)), coupled with the BLASTN results suggesting negligible Accumulibacter, 
520 the MiSeq data was interrogated in search of potential explanations. As described, Propionvibrio

521 was dominant among the phylotypes identified with the PAO primer set at the genus level, and it

522 may or may not be a PAO (Albertsen et al. 2016, Zhou et al. 2015). For the eubacterial primers,

523 Saccharibacteria spp. was prevalent across all operational days (also present in the Moscow

524 WRRF, but negligible in the other SBRs); these bacteria are part of the filamentous group of

525 microorganisms referred to as TM7 (Jenkins et al. 2003). Thiobacillus - a Betaproteobacteria-

526 was also consistently prominent in the G-EBPR MMC; this bacterium has the ability to store

527 glycogen and could be implicated in EBPR (it was also present at $10.8 \%$ and $13.8 \%$ on average

528 in V-and R-EBPR, respectively). Similar to Saccharibacteria spp., Haliscomenobacter (a

529 Bacteroidetes) is a filamentous bacterium common to activated sludge (Jenkins et al. 2003),

530 although it has been suggested that the bacterium cannot utilize glycerol (Kämpfer 1995); similar

531 enrichments were observed for this microorganism in all systems evaluated. Zoogloea (a

532 Betaproteobacteria) are viewed as critical to floc formation in activated sludge (Wen et al.

533 2015), and are common in activated sludge (Liu et al. 2016); Zoogloea was also enriched in V-

534 and R-EBPR. Of particular interest was the large fraction of Defluviicoccus, a putative GAO

535 (Carvalheira et al. 2014). However, being an Alphaproteobacteria, Defluviicoccus is capable of

536 PHA synthesis, and research has suggested certain clusters can store polyP (Wang et al. 2014). In

537 fact, Defluviicoccus was also relatively prominent in S-EBPR and to a lesser degree in V-EBPR.

538 Finally, Methylobacillus was relatively prominent in G-EBPR. Methylobacillus is a methanol

539 oxidizer, but there is no known linkage to EBPR.

540 Regarding other bacteria potentially important to EBPR, Tu and Schuler (2013), at times,

541 observed a relatively large fraction of Dechloromonas in a synthetic-fed EBPR system (and

542 suggested the potential for associated PAO behavior). Oehmen et al. (2010) similarly suggested 
543 Dechloromonas might be a PAO. However, this bacterium was only present on average less than

$5440.72 \%$ in MMCs this study. Azospira (within the Betaproteobacteria order) was detected in S-

545 EBPR using the PAO primers, however, this bacterium was minimally present within the

546 eubacterial dataset, suggesting no real consequence to EBPR (or at least in systems treating real

547 wastewater). Considering PHA production potential, and thus putative involvement with EBPR,

548 Thauera (within the Betaproteobacteria order; a known PHA producer (Lemos et al. 2008)) was

549 relatively prominent in S-, V-, and R-EBPR (>1\% on average; Table S49), but <1\% in G-EBPR

550 and the Moscow WRRF. While Meganema (an Alphaproteobacterium capable of excess PHA

551 synthesis (Hanson et al. 2016b)) was, on average, present in large numbers on average in V-, G-,

552 and R-EBPR, the average was skewed due to a large fraction observed in the MMC on

553 operational day 283.

$554 \quad$ Tetrasphaera are also linked to EBPR as putative PAOs (Nguyen et al. 2011, Nielsen et al.

555 2012). Being of the Actinobacteria phylum, the PAO primers used herein would not have

556 amplified Tetrasphaera; however, the genus was identified in the Eubacterial data set. Of

557 particular note, G-EBPR generally had the highest relative abundance of Tetrasphaera of the

558 systems evaluated (Fig. 4), with values approaching parity with Propionivibrio. Tetrasphaera

559 may be able to consume glycerol anaerobically, given the recently identified glycerol kinase in a

560 Tetrasphaera species (UniProt Accession: A0A0Q9M5T8) and the genus' ability to consume

561 glucose anaerobically (Nguyen et al. 2011). Additionally, the inability of Tetrasphaera to

562 process VFAs larger than acetate (Kong et al. 2005, Nguyen et al. 2011) could explain the

563 temporary EBPR failure observed by Coats et al. (2015) when the G-EBPR substrate was

564 switched from crude glycerol to VFA-rich primary solids fermentate. Accordingly, Tetrasphaera

565 could have contributed to the unexpected EBPR success in G-EBPR. 
Theory would suggest that high \%PAOs within the MMC is necessary for successful EBPR; such theory is backed up by a dominant array of synthetic wastewater-based research.

569 However, in this study attempts to relate the \%PAOs with the process performance metrics

570 proved unsuccessful. For all SBRs across all operational days, there was no apparent correlation

571 between \%PAO and effluent phosphorus or \%P removal (Tables 1, 3). Across the SBRs the

572 highest vs. lowest observed effluent P (and associated \%PAO) were $2.20 \mathrm{mg} \mathrm{P} \mathrm{L}^{-1}$ (4.48\%; S-

573 EBPR, day 86) and $0.03 \mathrm{mg} \mathrm{P} \mathrm{L}^{-1}$ (0.40\%; V-EBPR, day 86). Moreover, the lowest observed

$574 \%$ PAO (0.11\%; R-EBPR, day 263) exhibited good P removal (76.6\%). Indeed, the PAO fraction

575 in the real wastewater lab-scale systems was estimated to be much lower than observed in the

576 synthetic-fed SBR, yet overall P removal performance (excluding R-EBPR) was comparable.

577 The PAO population in the full-scale EBPR system, which achieved excellent P removal, was

578 quantitatively comparable to that of S-EBPR and consistently higher than observed in the real

579 wastewater-based SBRs, but again no correlation could be inferred. Finally, a comparative

580 analysis between the process metrics (VFA:P; P:C) and the qPCR PAO data revealed no

581 correlations (data not shown). Collectively, these comparative analyses suggest that EBPR

582 function did not follow with enrichment of PAOs. Indeed, Mao et al. (2015) and Liu et al. (2016)

583 observed numerous full-scale systems enriched with Accumulibacter that were not operated for

584 EBPR.

\section{4. Conclusions}

586 Research was conducted on lab- and full-scale EBPR systems, with a coupled examination

587 of MMC structure (applying qPCR and Illumina MiSeq analysis) and EBPR function;

588 investigations further considered synthetic vs. real wastewater. A central question throughout the 
study was the relative significance, or lack thereof, in the enrichment of a specific population of

590 PAOs in accomplishing excess P removal. Collectively, results suggest that induced EBPR

591 function transcends enrichment of any specific MMC population. While a strong relationship

592 was demonstrated between EBPR metrics (P:C; influent VFA:P) and excellent P removal across

593 diverse EBPR systems and MMCs, no such correlation existed with the specific MMCs (either

594 based on qPCR or MiSeq data). Multivariate analysis of the MiSeq-described MMCs revealed

595 distinct clusters based on substrate, with the synthetic wastewater fed MMC unique from that of

596 the real wastewater systems, including a full-scale EBPR system. qPCR results based on primers

597 designed for the putative PAO Accumulibacter did not correlate with BLASTN eubacterial

598 results for either Accumulibacter or Rhodocyclaceae. More critically, RDP assignment of PAO-

599 based sequences aligned poorly with Accumulibacter for both eubacterial and PAO primer sets,

600 which strongly suggests that the conventional PAO primers applied in FISH and qPCR analysis

601 do not sufficiently target the putative PAO Accumulibacter. In particular, negligible alignment

602 was observed for PAO amplicons obtained from a MMC performing excellent EBPR on crude

603 glycerol (an atypical substrate). The best observed BLASTN match of the PAO amplicons was

604 for a synthetic wastewater-based MMC; this result raises concerns about the potential relevance

605 in using synthetic substrates in the study of EBPR.

606

607 Acknowledgements

608 The material presented and discussed herein is based upon work supported by i) an

609 Institutional Development Award (IDeA) from the National Institute of General Medical

610 Sciences of the National Institutes of Health under Grant Number P20GM103408 and ii) the

611 National Science Foundation under Grant Number CBET-1235885. Any opinions, findings, and 
612 conclusions or recommendations expressed in this material are those of the authors and do not

613 necessarily reflect the views of the funding agencies.

614 The authors greatly appreciate the efforts of Nicholas M. Guho, who prepared and

615 executed workflows to evaluate the MiSeq results and generate the associated tables and figures. 
Figure 1 - Influent wastewater P:C ratio against effluent P for an array of laboratory-scale EBPR reactors (this study and (Coats et al. 2011a, Coats et al. 2011b, Coats et al. 2015, Horgan

619 et al. 2010, Winkler et al. 2011)).

Figure 2 - Influent wastewater VFA:P ratio against effluent P for an array of laboratory-

621 scale EBPR reactors (this study and (Coats et al. 2011a, Coats et al. 2011b, Coats et al. 2015,

622 Horgan et al. 2010, Winkler et al. 2011)).

Figure 3 - Bivariate plot of the first two principal component (Dim 1 and Dim 2) scores for

624 the 16S rRNA gene sequencing results using the EUB primer set at the genus level and the $\mathrm{P}$ 625 removed. Samples have been grouped by system (distinguished by color) with the numbers 626 corresponding to their index in an ordered list (i.e., sorted by operational day following an 627 arbitrary system ordering: 1 to 7 are S-EBPR operational days 72 to $283 ; 8$ to 14 are V-EBPR 628 operational days 72 to $283 ; 15$ to 21 are G-EBPR operational days 72 to 283 ; and 22 to 28 are R-

629 EBPR operational days 72 to 283. The centroid for each group in the Dim 1-Dim 2 space is

630 depicted as a square in the associated color and the ellipses denote the 95\% confidence regions

631 for the associated centroids in the Dim 1-Dim 2 space.

Figure 4 - Heat map showing the relative abundance of phylotypes identified at the genus

633 level using the EUB primer set. Unidentified phylotypes (denoted as "unknown") were

634 aggregated by the most specific taxonomic level at which they were identified (the "**” is used to 635 indicate any identified taxon name). Note that the adopted color scheme is nonlinear at $0 \%$

636 relative abundance to differentiate phylotypes which were not detected (N. D.) in a sample from

637 those that were. To conserve space, some taxon names have been abbreviated (Ca.: Candidatus;

$638 \quad$ a $:$ incertae_sedis; ${ }^{\mathrm{b}}$ :_genera_incertae_sedis; and ${ }^{\mathrm{c}}$ : sensu stricto). The phylotypes with less than 
$6392.5 \%$ relative abundance in all samples were aggregated and denoted "Phylotypes $<2.5 \%$ "

640 followed by the number of phylotypes so categorized in parentheses at the bottom of the heat 641 map.

642 


\section{LIST OF TABLES}

644 Table 1 - Performance characteristics for each SBR and Moscow WRRF, by operational 645 day, including influent/effluent phosphorus and \% P removal. Influent wastewater VFA:P ratio, 646 phosphorus released anaerobically, and the anaerobic P:C ratio summarized for the SBRs.

647 Table 2 - Performance summary for the bench-scale EBPR reactors.

648 Table 3 - Results from qPCR applied to DNA extracted from MLSS obtained from each 649 SBR and the Moscow WRRF. Data shown are composite averages/standard deviation for each 650 operational day.

651 Table 4 - Major and minor bacterial community composition at the phylum level (based on 652 the calculated averages across all operational days evaluated; listed in order of dominance) 653 detected for Eubacteria, PAOs, and GAOs in the lab- and full-scale EBPR WRRFs.

654 Table 5 - Major and minor bacterial community composition at the order level (based on 655 the calculated averages across all operational days evaluated; listed in order of dominance) 656 detected for Eubacteria, PAOs, and GAOs in the lab- and full-scale EBPR WRRFs. 
Al-Najjar, M.M., Coats, E.R. and Loge, F.J. (2011) The role of the microbial stringent response in excess intracellular accumulation of phosphorous in mixed consortia fed synthetic wastewater. Water Res. 45(16), 5038-5046.

Albertsen, M., Hansen, L.B.S., Saunders, A.M., Nielsen, P.H. and Nielsen, K.L. (2012) A metagenome of a full-scale microbial community carrying out enhanced biological phosphorus removal. The ISME Journal 6(6), 1094-1106.

Albertsen, M., McIlroy, S.J., Stokholm-Bjerregaard, M., Karst, S.M. and Nielsen, P.H. (2016) "Candidatus Propionivibrio aalborgensis": a novel glycogen accumulating organism abundant in full-scale enhanced biological phosphorus removal plants. Frontiers in Microbiology 7.

Bond, P.L., Hugenholtz, P., Keller, J. and Blackall, L.L. (1995) Bacterial community structures of phosphate-removing and non-phosphate-removing activated sludges from sequencing batch reactors. Appl. Environ. Microb. 61(5), 1910-1916.

Bond, P.L., Erhart, R., Wagner, M., Keller, J. and Blackall, L.L. (1999) Identification of some of the major groups of bacteria in efficient and nonefficient biological phosphorus removal activated sludge systems. Appl. Environ. Microbiol. 65(9), 4077-4084.

Brooks, B.W., Lazorchak, J.M., Howard, M.D.A., Johnson, M.-V.V., Morton, S.L., Perkins, D.A.K., Reavie, E.D., Scott, G.I., Smith, S.A. and Steevens, J.A. (2016) Are harmful algal blooms becoming the greatest inland water quality threat to public health and aquatic ecosystems? Environmental Toxicology and Chemistry 35(1), 6-13.

Camacho, C., Coulouris, G., Avagyan, V., Ma, N., Papadopoulos, J., Bealer, K. and Madden, T.L. (2009) BLAST+: architecture and applications. BMC Bioinformatics 10(1), 1-9.

Carvalheira, M., Oehmen, A., Carvalho, G. and Reis, M.A.M. (2014) The effect of substrate competition on the metabolism of polyphosphate accumulating organisms (PAOs). Water Res. 64, 149-159.

Coats, E.R., Mockos, A. and Loge, F.J. (2011a) Post-anoxic denitrification driven by PHA and glycogen within enhanced biological phosphorus removal. Bioresour. Technol. 102, 1019-1027.

Coats, E.R., Watkins, D.L., Brinkman, C.K. and Loge, F.J. (2011b) Effect of anaerobic HRT on biological phosphorus removal and the enrichment of phosphorus accumulating organisms. Water Environ. Res. 83(5), 461-469.

Coats, E.R., Watkins, D.L. and Kranenburg, D. (2011c) A comprative environmental life cycle analysis for removing phosphorus from wastewater: Biological versus physical/chemical processes. Water Environ. Res. 83(8), 750-760.

Coats, E.R., Dobroth, Z.T. and Brinkman, C.K. (2015) EBPR using crude glycerol: Assessing process resiliency and exploring metabolic anomalies. Water Environ. Res. 87(1), 68-79.

Crocetti, G.R., Hugenholtz, P., Bond, P.L., Schuler, A., Keller, J., Jenkins, D. and Blackall, L.L. (2000) Identification of polyphosphate-accumulating organisms and design of $16 \mathrm{~S}$ rRNA-directed probes for their detection and quantitation. Appl. Environ. Microbiol. 66(3), 1175-1182.

Crocetti, G.R., Banfield, J.F., Keller, J., Bond, P.L. and Blackall, L.L. (2002) Glycogenaccumulating organisms in laboratory-scale and full-scale wastewater treatment processes. Microbiol. 148, 3353-3364. 
Filipe, C.D.M., Daigger, G.T. and Grady, C.P.L. (2001) Stoichiometry and kinetics of acetate uptake under anaerobic conditions by an enriched culture of phosphorus-accumulating organisms at different pHs. Biotechnol. Bioeng. 76(1), 32-43.

Garcia Martin, H., Ivanova, N., Kunin, V., Warnecke, F., Barry, K.W., McHardy, A.C., Yeates, C., He, S., Salamov, A.A., Szeto, E., Dalin, E., Putnam, N.H., Shapiro, H.J., Pangilinan, J.L., Rigoutsos, I., Kyrpides, N.C., Blackall, L.L., McMahon, K.D. and Hugenholtz, P. (2006) Metagenomic analysis of two enhanced biological phosphorus removal (EBPR) sludge communities. Nat. Biotechnol. 24(10), 1263-1269.

Grady Jr., C.P.L., Daigger, G.T., Love, N.G. and Filipe, C.D.M. (2011) Biological Wastewater Treatment, IWA Publishing/CRC Press, Boca Raton, FL.

Hanson, A.J., Guho, N.M., Paszczynski, A.J. and Coats, E.R. (2016a) Community proteomics provides functional insight into polyhydroxyalkanoate production by a mixed microbial culture cultivated on fermented dairy manure. Appl. Biochem. Biotechnol., 1-20.

Hanson, A.J., Paszczynski, A.J. and Coats, E.R. (2016b) Proteomic profiling of an undefined microbial consortium cultured in fermented dairy manure: Methods development. Electrophoresis 37(5-6), 790-794.

He, S., Gall, D.L. and McMahon, K.D. (2007) "Candidatus Accumulibacter" population structure in enhanced biological phosphorus removal sludges as revealed by polyphosphate kinase genes. Appl. Environ. Microbiol. 73(18), 5865-5874.

Heathwaite, L. and Sharpley, A. (1999) Evaluating measures to control the impact of agricultural phosphorus on water quality. Water Sci. Technol. 39(12), 149-155.

Hesselmann, R.P.X., Werlen, C., Hahn, D., van der Meer, J.R. and Zehnder, A.J.B. (1999) Enrichment, phylogenetic analysis and detection of a bacterium that performs enhanced biological phosphate removal in activated sludge. Syst. Appl. Microbiol. 22(3), 454-465.

Hill, T.C.J., Walsh, K.A., Harris, J.A. and Moffett, B.F. (2003) Using ecological diversity measures with bacterial communities. FEMS Microbiol Ecol 43(1), 1-11.

Horgan, C.J., Coats, E.R. and Loge, F.J. (2010) Assessing the effects of solids residence time and volatile fatty acid augmentation on biological phosphorus removal using real wastewater. Water Environ. Res. 82(3), 216-226.

Hu, M., Wang, X., Wen, X. and Xia, Y. (2012) Microbial community structures in different wastewater treatment plants as revealed by 454 -pyrosequencing analysis. Bioresour. Technol. 117, 72-79.

Jenkins, D., Richard, M.G. and Daigger, G.T. (2003) Manual on the causes and control of activated sludge bulking, foaming, and other solids separation problems, Lewis Publishers.

Kämpfer, P. (1995) Physiological and Chemotaxonomic Characterization of Filamentous Bacteria Belonging to the Genus Haliscomenobacter. Syst. Appl. Microbiol. 18(3), 363367.

Kim, B.-C., Kim, S., Shin, T., Kim, H. and Sang, B.-I. (2013) Comparison of the Bacterial Communities in Anaerobic, Anoxic, and Oxic Chambers of a Pilot A2O Process Using Pyrosequencing Analysis. Curr. Microbiol. 66(6), 555-565.

Kong, Y., Nielsen, J.L. and Nielsen, P.H. (2005) Identity and Ecophysiology of Uncultured Actinobacterial Polyphosphate-Accumulating Organisms in Full-Scale Enhanced Biological Phosphorus Removal Plants. Appl. Environ. Microbiol. 71(7), 4076-4085.

Lemos, P.C., Serafim, L.S., Santos, M.M., Reis, M.A.M. and Santos, H. (2003) Metabolic pathway for propionate utilization by phosphorus-accumulating organisms in activated 

69(1), 241-251.

Lemos, P.C., Levantesi, C., Serafim, L.S., Rossetti, S., Reis, M.A.M. and Tandoi, V. (2008) Microbial characterisation of polyhydroxyalkanoates storing populations selected under different operating conditions using a cell-sorting RT-PCR approach. Appl. Microbiol. Biotechnol. 78(2), 351-360.

Liu, T., Liu, S., Zheng, M., Chen, Q. and Ni, J. (2016) Performance Assessment of Full-Scale Wastewater Treatment Plants Based on Seasonal Variability of Microbial Communities via High-Throughput Sequencing. PLoS ONE 11(4), e0152998.

Lopez-Vazquez, C.M., Hooijmans, C.M., Brdjanovic, D., Gijzen, H.J. and van Loosdrecht, M.C.M. (2007) A practical method for quantification of phosphorus- and glycogenaccumulating organism populations in activated sludge systems. Water Environ. Res. 79(13), 2487-2498.

Lu, H., Oehmen, A., Virdis, B., Keller, J. and Yuan, Z. (2006) Obtaining highly enriched cultures of Candidatus Accumulibacter phosphates through alternating carbon sources. Water Res. 40, 3838-3848.

Magoč, T. and Salzberg, S.L. (2011) FLASH: fast length adjustment of short reads to improve genome assemblies. Bioinformatics 27(21), 2957-2963.

Mao, Y., Graham, D.W., Tamaki, H. and Zhang, T. (2015) Dominant and novel clades of Candidatus Accumulibacter phosphatis in 18 globally distributed full-scale wastewater treatment plants. Scientific Reports 5, 11857.

Muyzer, G., de Waal, E.C. and Uitterlinden, A.G. (1993) Profiling of complex microbial populations by denaturing gradient gel electrophoresis analysis of polymerase chain reaction-amplified genes coding for 16S rRNA. Appl. Environ. Microbiol. 59(3), 695700.

Nguyen, H.T.T., Le, V.Q., Hansen, A.A., Nielsen, J.L. and Nielsen, P.H. (2011) High diversity and abundance of putative polyphosphate-accumulating Tetrasphaera-related bacteria in activated sludge systems. FEMS Microbiology Ecology 76(2), 256-267.

Nguyen, H.T.T., Nielsen, J.L. and Nielsen, P.H. (2012) 'Candidatus Halomonas phosphatis', a novel polyphosphate-accumulating organism in full-scale enhanced biological phosphorus removal plants. Environ. Microbiol. 14(10), 2826-2837.

Nielsen, P.H., Mielczarek, A.T., Kragelund, C., Nielsen, J.L., Saunders, A.M., Kong, Y., Hansen, A.A. and Vollertsen, J. (2010) A conceptual ecosystem model of microbial communities in enhanced biological phosphorus removal plants. Water Res. 44(17), 5070-5088.

Nielsen, P.H., Saunders, A.M., Hansen, A.A., Larsen, P. and Nielsen, J.L. (2012) Microbial communities involved in enhanced biological phosphorus removal from wastewater - a model system in environmental biotechnology. Current Opinion in Biotechnology 23(3), 452-459.

Oehmen, A., Lemos, P.C., Carvalho, G., Yuan, Z.G., Keller, J., Blackall, L.L. and Reis, M.A.M. (2007) Advances in enhanced biological phosphorus removal: From micro to macro scale. Water Res. 41(11), 2271-2300.

Oehmen, A., Carvalho, G., Lopez-Vazquez, C.M., van Loosdrecht, M.C.M. and Reis, M.A.M. (2010) Incorporating microbial ecology into the metabolic modelling of polyphosphate accumulating organisms and glycogen accumulating organisms. Water Res. 44, 49925004. 
Paerl, H. (2014) Mitigating Harmful Cyanobacterial Blooms in a Human- and ClimaticallyImpacted World. Life 4(4), 988.

Pretty, J.N., Mason, C.F., Nedwell, D.B., Hine, R.E., Leak, S. and Dils, R. (2003) Environmental costs of freshwater eutrophication in England and Wales. Environ. Sci. Technol. 37(2), 201-208.

Ramakers, C., Ruijter, J.M., Deprez, R.H.L. and Moorman, A.F.M. (2003) Assumption-free analysis of quantitative real-time polymerase chain reaction (PCR) data. Neuroscience Letters 339(1), 62-66.

Seviour, R.J., Mino, T. and Onuki, M. (2003) The microbiology of biological phosphorus removal in activated sludge systems. FEMS Microbiol. Rev. 27, 99-127.

Shen, J., Song, N., Williams, C.J., Brown, C.J., Yan, Z., Xu, C. and Forney, L.J. (2016) Effects of low dose estrogen therapy on the vaginal microbiomes of women with atrophic vaginitis. Scientific Reports 6, 24380.

Smolders, G.J.F., van der Meij, J., van Loosdrecht, M.C.M. and Heijnen, J.J. (1994) Model of the anaerobic metabolism of the biological phosphorus removal process: stoichiometry and $\mathrm{pH}$ influence. Biotechnol. Bioeng. 43(6), 461-470.

Thompson, J.C. and He, B.B. (2006) Characterization of crude glycerol from biodiesel production from multiple feedstocks. Appl. Eng. Agric. 22(2), 261-265.

Tu, Y. and Schuler, A.J. (2013) Low Acetate Concentrations Favor PolyphosphateAccumulating Organisms over Glycogen-Accumulating Organisms in Enhanced Biological Phosphorus Removal from Wastewater. Environ. Sci. Technol. 47(8), 38163824.

Wang, Q., Garrity, G.M., Tiedje, J.M. and Cole, J.R. (2007) Naïve Bayesian classifier for rapid assignment of rRNA sequences into the New Bacterial Taxonomy. Appl Environ Microbiol 73(16), 5261-5267.

Wang, X., Hu, M., Xia, Y., Wen, X. and Ding, K. (2012) Pyrosequencing Analysis of Bacterial Diversity in 14 Wastewater Treatment Systems in China. Appl. Environ. Microb. 78(19), 7042-7047.

Wang, Z., Guo, F., Mao, Y., Xia, Y. and Zhang, T. (2014) Metabolic Characteristics of a Glycogen-Accumulating Organism in Defluviicoccus Cluster II Revealed by Comparative Genomics. Microbial Ecol. 68(4), 716-728.

WEF (2010) Nutrient Removal: Manual of Practice No. 34, WEF Press, Alexandria, Virginia.

Wen, Y., Jin, Y., Wang, J. and Cai, L. (2015) MiSeq sequencing analysis of bacterial community structures in wastewater treatment plants. Pol. J. Environ. Stud. 24(4), 1809-1815.

Winkler, M., Coats, E.R. and Brinkman, C.K. (2011) Advancing post-anoxic denitrification for biological nutrient removal. Water Res. 45(18), 6119-6130.

Zhang, H., Sekiguchi, Y., Hanada, S., Hugenholtz, P., Kim, H., Kamagata, Y. and Nakamura, K. (2003) Gemmatimonas aurantiaca gen. nov., sp. nov., a Gram-negative, aerobic, polyphosphate-accumulating micro-organism, the first cultured representative of the new bacterial phylum Gemmatimonadetes phyl. nov. International Journal of Systematic and Evolutionary Microbiology 53(4), 1155-1163.

Zhou, Z., Qiao, W., Xing, C., An, Y., Shen, X., Ren, W., Jiang, L.-m. and Wang, L. (2015) Microbial community structure of anoxic-oxic-settling-anaerobic sludge reduction process revealed by 454-pyrosequencing. Chemical Engineering Journal 266, 249-257. 


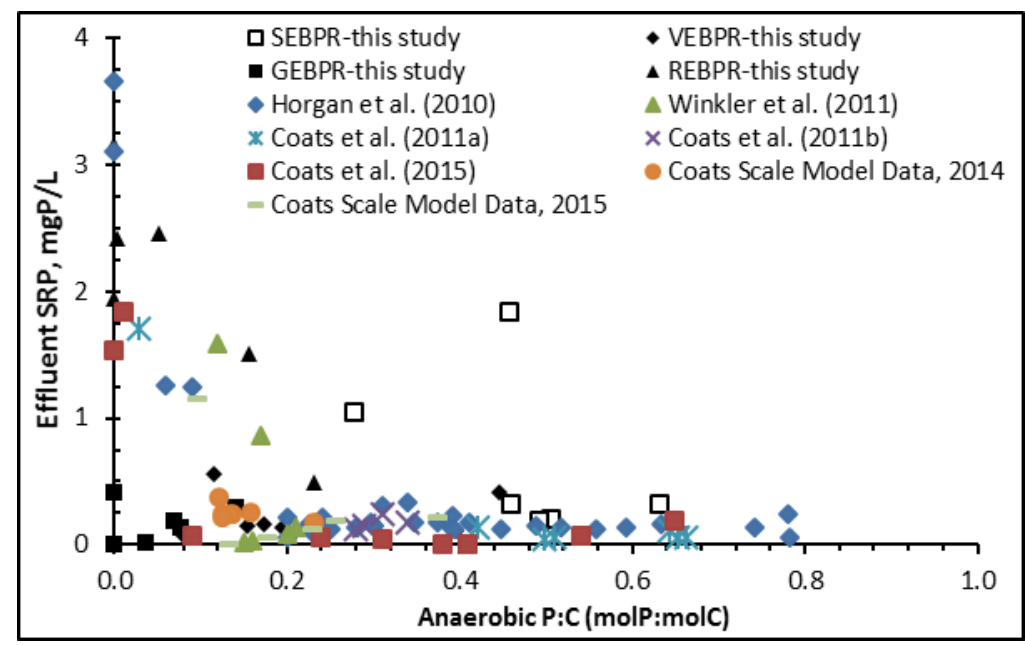




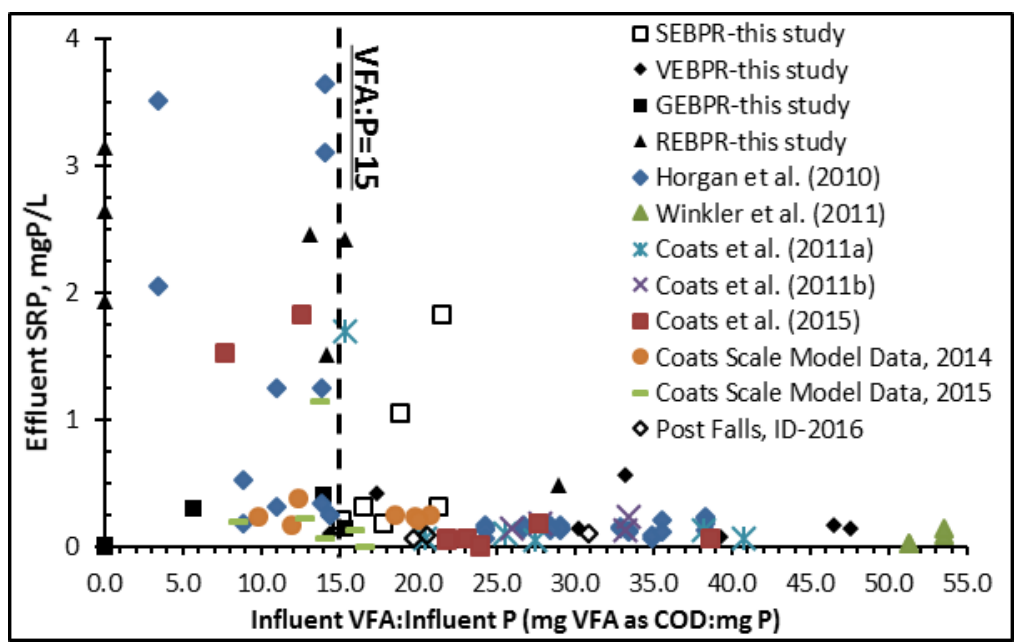




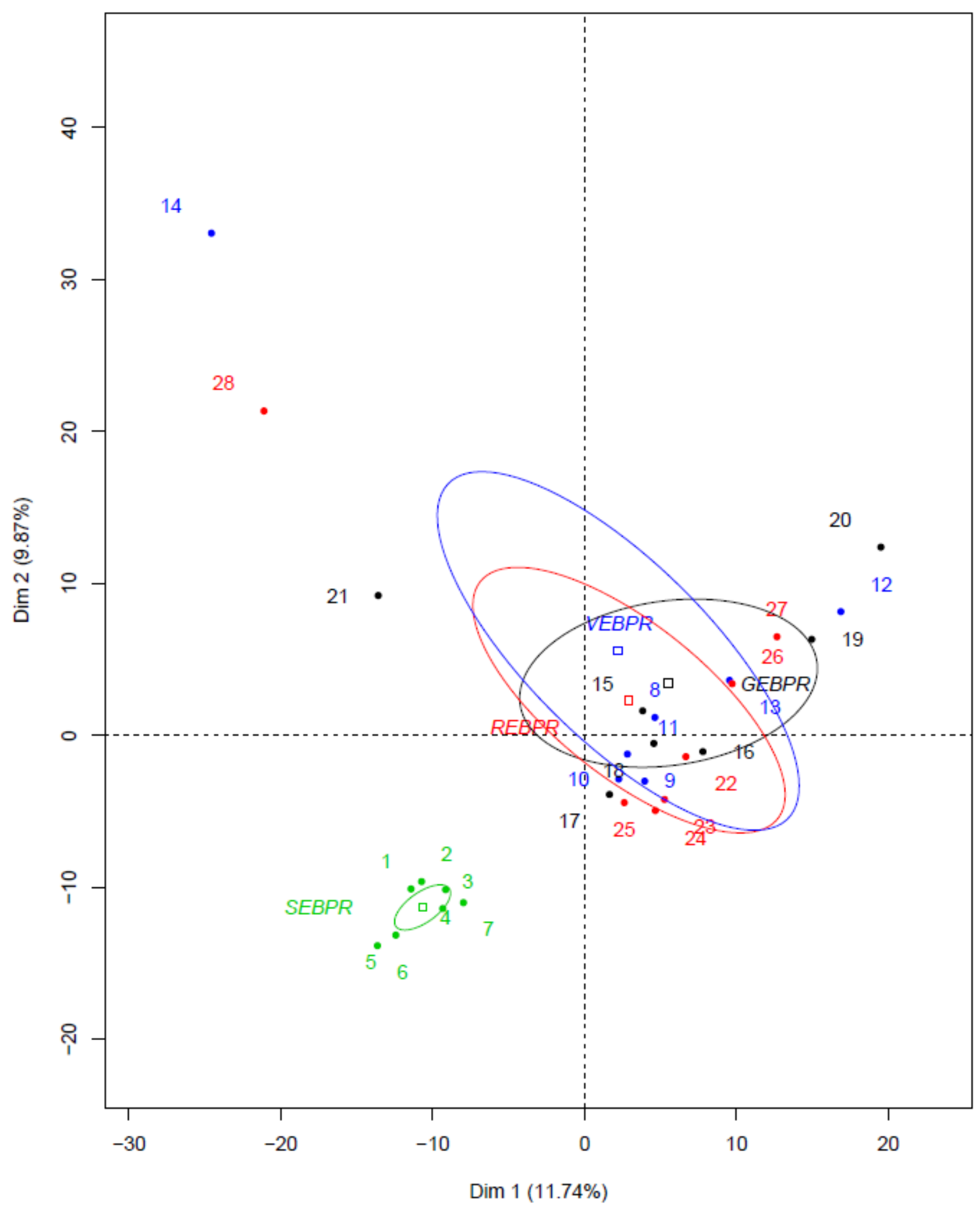

Figure 3 


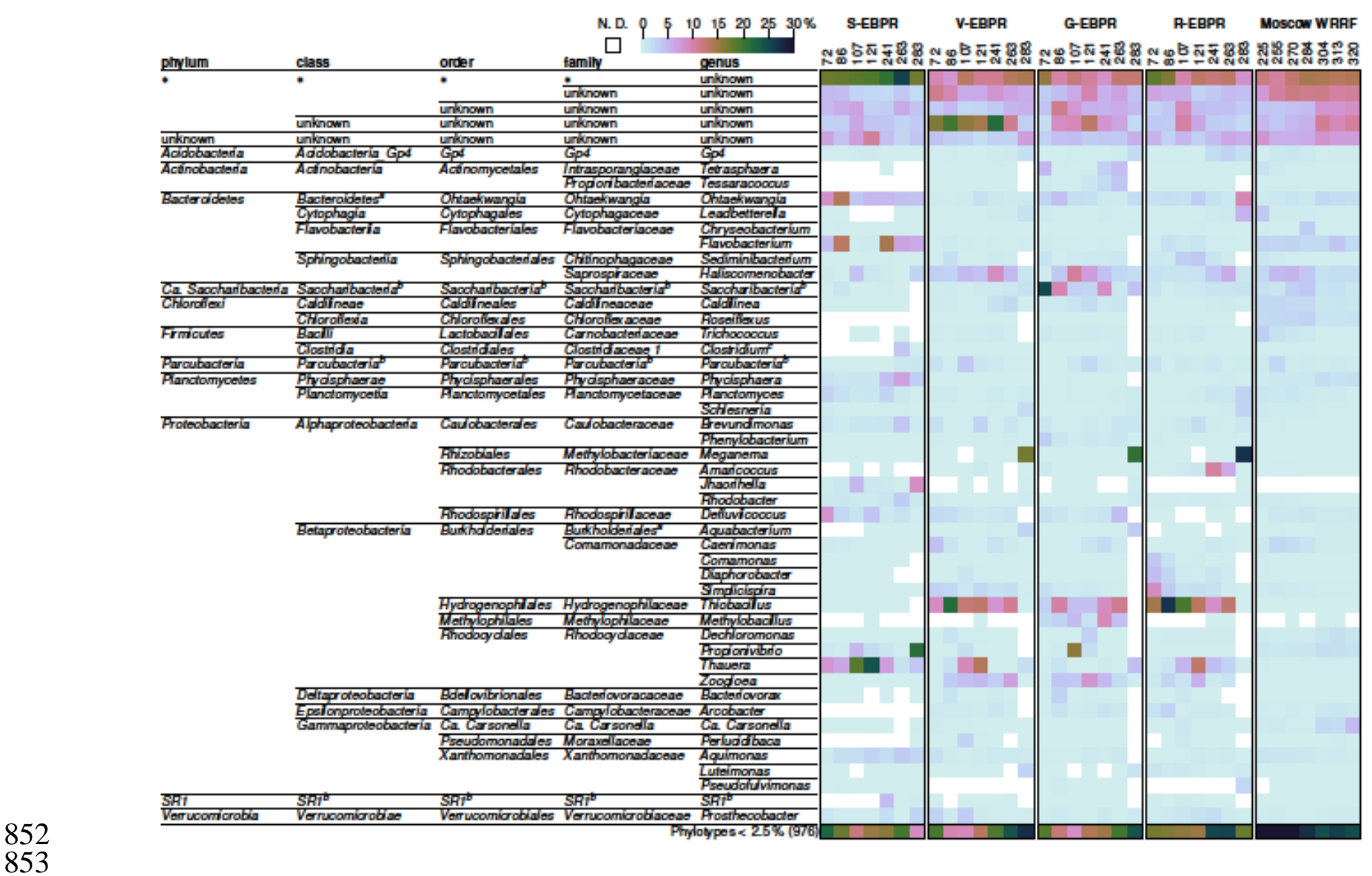


Table 1

\begin{tabular}{|c|c|c|c|c|c|c|c|}
\hline & Op'l Day & $\begin{array}{l}\text { Infl. P, } \\
\left(\mathrm{mg} \mathrm{L}^{-1}\right)\end{array}$ & $\begin{array}{l}\text { Effl. P, } \\
\left(\mathrm{mg} \mathrm{L} \mathrm{L}^{-1}\right)\end{array}$ & $\begin{array}{c}\% \mathbf{P} \\
\text { Removal }\end{array}$ & $\begin{array}{c}\text { Infl. VFA:P } \\
\left(\mathrm{mg} \text { COD mg } \mathbf{P}^{-1}\right)\end{array}$ & $\begin{array}{c}\mathbf{P}_{\text {rel }} \\
\left(\mathrm{mg} \mathrm{L}^{-1}\right)\end{array}$ & $\begin{array}{c}\text { P:C } \\
\left(\text { Pmol Cmol }^{-1}\right)\end{array}$ \\
\hline \multirow{7}{*}{$\begin{array}{l}\frac{1}{2} \\
0 \\
\text { un } \\
\text { is }\end{array}$} & 72 & 15.14 & 0.25 & 98.3 & 39.9 & 87.9 & 0.512 \\
\hline & 86 & 19.31 & 2.20 & 88.6 & 31.8 & 46.9 & -0.149 \\
\hline & 107 & 18.82 & 1.08 & 94.3 & 33.6 & 53 & 0.284 \\
\hline & 121 & 21.54 & 1.86 & 91.4 & 18.5 & 54.5 & 0.467 \\
\hline & 241 & 16.48 & 0.35 & 97.9 & 30.2 & 69.1 & 0.469 \\
\hline & 263 & 17.83 & 0.30 & 98.3 & 33.0 & 92.3 & 0.526 \\
\hline & 283 & 21.31 & 0.44 & 97.9 & 20.0 & 85.2 & 0.679 \\
\hline \multirow{7}{*}{$\begin{array}{l}\frac{\alpha}{2} \\
0 \\
11 \\
>^{\prime}\end{array}$} & 72 & 5.49 & 0.23 & 95.8 & 46.5 & 12.3 & 0.18 \\
\hline & 86 & 6.17 & 0.03 & 99.5 & 30.3 & 9.4 & 0.172 \\
\hline & 107 & 5.72 & 0.59 & 89.7 & 39.3 & 6.1 & 0.094 \\
\hline & 121 & 6.59 & 0.10 & 98.5 & 33.2 & 8 & 0.13 \\
\hline & 241 & 8.83 & 0.12 & 98.6 & 14.4 & 15.6 & 0.423 \\
\hline & 263 & 4.40 & 0.25 & 94.3 & 47.5 & 12 & 0.197 \\
\hline & 283 & 5.73 & 0.54 & 90.6 & 17.4 & 13.5 & 0.452 \\
\hline \multirow{7}{*}{$\begin{array}{l}\frac{0}{\Delta} \\
\frac{1}{4} \\
0\end{array}$} & 72 & 3.04 & 0.17 & 94.4 & 15.4 & 1.12 & 0.079 \\
\hline & 86 & 3.43 & 0.04 & 98.8 & 0.0 & -0.97 & No VFAs \\
\hline & 107 & 2.58 & 0.23 & 91.1 & 13.1 & 0.82 & 0.08 \\
\hline & 121 & 3.49 & 0.04 & 98.9 & 0.0 & 0.55 & no VFAs \\
\hline & 241 & 4.50 & 0.44 & 90.2 & 14.0 & -0.31 & -0.02 \\
\hline & 263 & 3.22 & 0.13 & 95.7 & 24.1 & 1.18 & 0.05 \\
\hline & 283 & 4.86 & 0.41 & 91.6 & 5.6 & 1.36 & 0.181 \\
\hline \multirow{7}{*}{\begin{tabular}{l}
$\frac{a}{a}$ \\
\multicolumn{1}{|c}{} \\
$\stackrel{1}{\alpha}$
\end{tabular}} & 72 & 3.04 & 2.47 & 18.8 & 15.4 & 0.06 & 0.004 \\
\hline & 86 & 3.45 & 2.67 & 22.6 & 0.0 & 0 & no VFAs \\
\hline & 107 & 2.58 & 2.49 & 3.5 & 13.1 & 0.64 & 0.063 \\
\hline & 121 & 3.45 & 1.96 & 43.2 & 0.0 & 1.02 & no VFAs \\
\hline & 241 & 4.34 & 1.56 & 64.1 & 14.1 & 2.78 & 0.156 \\
\hline & 263 & 2.91 & 0.68 & 76.6 & 29.0 & 6.24 & 0.243 \\
\hline & 283 & 3.49 & 3.35 & 4.0 & 0.0 & 2.29 & no VFAs \\
\hline \multirow{7}{*}{$\begin{array}{l}\frac{1}{\alpha} \\
\mathbf{n} \\
3 \\
0 \\
0 \\
0 \\
\Sigma \\
\Sigma\end{array}$} & 225 & 5.01 & 0.15 & 97.0 & -- & -- & -- \\
\hline & 255 & 4.42 & 0.39 & 91.2 & -- & -- & -- \\
\hline & 270 & 4.76 & 0.20 & 95.9 & -- & -- & -- \\
\hline & 284 & 4.59 & 0.16 & 96.5 & -- & -- & -- \\
\hline & 304 & 4.70 & $<0.05$ & $>98.9$ & -- & -- & -- \\
\hline & 313 & 4.99 & $<0.05$ & $>99.0$ & -- & -- & -- \\
\hline & 320 & 5.71 & $<0.05$ & $>99.1$ & -- & -- & -- \\
\hline
\end{tabular}


Table 2

\begin{tabular}{|c|c|c|c|c|c|c|c|}
\hline Parameter & Units & Reactor & Average & Maximum & Minimum & SD & $\mathbf{n}$ \\
\hline \multirow{4}{*}{ MLSS } & \multirow{4}{*}{$\mathrm{mg} / \mathrm{L}$} & S-EBPR & 3888 & 6000 & 1530 & 1122 & 23 \\
\hline & & V-EBPR & 2631 & 3680 & 1740 & 632 & 23 \\
\hline & & G-EBPR & 2177 & 3040 & 780 & 598 & 18 \\
\hline & & R-EBPR & 1547 & 2500 & 900 & 435 & 12 \\
\hline \multirow{4}{*}{ Influent Phosphorus } & \multirow{4}{*}{$\mathrm{mgP} / \mathrm{L}$} & S-EBPR & 18.11 & 27.80 & 12.98 & 3.08 & 63 \\
\hline & & V-EBPR & 5.68 & 8.24 & 3.76 & 0.98 & 64 \\
\hline & & G-EBPR & 4.13 & 5.32 & 2.51 & 0.85 & 28 \\
\hline & & R-EBPR & 3.97 & 5.51 & 2.34 & 0.95 & 28 \\
\hline \multirow{4}{*}{ Effluent Phosphorus } & \multirow{4}{*}{$\mathrm{mgP} / \mathrm{L}$} & S-EBPR & $1.75^{1}$ & $47.87^{1}$ & 0.01 & 5.47 & 136 \\
\hline & & V-EBPR & $0.73^{2}$ & $7.06^{2}$ & 0.00 & 1.31 & 131 \\
\hline & & G-EBPR & $0.29^{2}$ & $3.59^{3}$ & 0.00 & 0.50 & 85 \\
\hline & & R-EBPR & 2.03 & 7.54 & 0.12 & 1.64 & 82 \\
\hline \multirow{4}{*}{$\begin{array}{c}\text { Influent } \\
\text { VFA:Influent P }\end{array}$} & \multirow{4}{*}{$\begin{array}{l}\text { mgVFA (as } \\
\text { COD)/mgP }\end{array}$} & S-EBPR & 31.85 & 43.95 & 19.66 & 7.53 & 14 \\
\hline & & V-EBPR & 33.34 & 49.23 & 15.47 & 11.34 & 11 \\
\hline & & G-EBPR & 12.35 & 29.32 & 0.00 & 9.96 & 8 \\
\hline & & R-EBPR & 12.41 & 36.05 & 0.00 & 12.46 & 8 \\
\hline
\end{tabular}

${ }^{1}$ S-EBPR operations realized a temporary upset associated with misfeeding of synthetic substrate. Excluding approximately 30 days of operational instability, the maximum effluent S-EBPR phosphorus was $9.6 \mathrm{mgP} / \mathrm{L}$, while the average was $0.68 \mathrm{mgP} / \mathrm{L}$.

${ }^{2}$ Excluding 21 days that were deemed process upsets, the maximum effluent F-EBPR phosphorus was $0.92 \mathrm{mgP} / \mathrm{L}$, while the average was $0.23 \mathrm{mgP} / \mathrm{L}$.

${ }^{3}$ Excluding two observed days of process upset, the maximum effluent G-EBPR phosphorus was $0.54 \mathrm{mgP} / \mathrm{L}$, while the average was $0.21 \mathrm{mgP} / \mathrm{L}$.

${ }^{4}$ On several occasions both the G- and R-EBPR influent contained no VFAs, which is consistent with previous observations (Coats et al. 2015). 
Table 3

\begin{tabular}{|c|c|c|c|c|c|c|c|c|c|c|c|}
\hline \multirow[b]{2}{*}{$\begin{array}{l}\text { Op'l } \\
\text { Day }\end{array}$} & \multicolumn{2}{|c|}{ S-EBPR } & \multicolumn{2}{|c|}{ V-EBPR } & \multicolumn{2}{|c|}{ G-EBPR } & \multicolumn{2}{|c|}{ R-EBPR } & \multirow[b]{2}{*}{$\begin{array}{l}\text { Op'l } \\
\text { Day }\end{array}$} & \multicolumn{2}{|c|}{ Moscow WRRF } \\
\hline & $\begin{array}{c}\% \text { PAO } \\
\operatorname{avg}(\text { SD) }\end{array}$ & $\begin{array}{r}\% \text { GAO } \\
\text { avg (SD) }\end{array}$ & $\begin{array}{r}\% \text { PAO } \\
\operatorname{avg}(\mathrm{SD}) \\
\end{array}$ & $\begin{array}{r}\% \text { GAO } \\
\operatorname{avg}(\mathrm{SD}) \\
\end{array}$ & $\begin{array}{c}\% \text { PAO } \\
\text { avg (SD) }\end{array}$ & $\begin{array}{c}\% \text { GAO } \\
\operatorname{avg}(\mathrm{SD})\end{array}$ & $\begin{array}{c}\% \text { PAO } \\
\operatorname{avg}(\mathrm{SD})\end{array}$ & $\begin{array}{c}\% \text { GAO } \\
\operatorname{avg}(\mathrm{SD}) \\
\end{array}$ & & $\begin{array}{c}\% \text { PAO } \\
\text { avg (SD) }\end{array}$ & $\begin{array}{c}\% \text { GAO } \\
\operatorname{avg}(\mathrm{SD})\end{array}$ \\
\hline 72 & $4.17(0.53)$ & $0.21(0.09)$ & $0.67(0.13)$ & $0.45(0.19)$ & $0.40(0.14)$ & $0.05(0.02)$ & $0.52(0.12$ & $0.04(0.03)$ & 225 & $3.97(0.11)$ & $0.36(0.01)$ \\
\hline 86 & $4.48(0.63)$ & $0.35(0.17)$ & $0.40(0.07)$ & $1.78(0.63)$ & $0.27(0.03)$ & $0.14(0.07)$ & $1.14(0.27)$ & $0.02(0.01)$ & 255 & $7.23(0.30)$ & $0.46(0.03)$ \\
\hline 107 & 4.09 (1.19) & $0.54(0.34)$ & $0.12(0.03)$ & $0.04(0.02)$ & $0.26(0.06)$ & $0.23(0.11)$ & $0.62(0.14)$ & $0.01(0.01)$ & 270 & $5.33(0.59)$ & $0.27(0.02)$ \\
\hline 121 & $2.92(1.37)$ & $0.06(0.02)$ & $0.24(0.12)$ & $0.14(0.03)$ & $0.32(0.07)$ & $0.48(0.23)$ & $0.17(0.05)$ & $0.04(0.03)$ & 284 & $15.46(0.46)$ & $0.46(0.05)$ \\
\hline 241 & $5.42(2.21)$ & $0.07(0.03)$ & $0.21(0.08)$ & $1.18(0.61)$ & $0.42(0.17)$ & $0.40(0.19)$ & $0.16(0.04)$ & $0.00(0.00)$ & 304 & $9.09(0.79)$ & $0.41(0.02)$ \\
\hline 263 & $0.61(0.11)$ & $0.04(0.02)$ & $0.42(0.15)$ & $1.21(0.58)$ & $0.79(0.29)$ & $0.45(1.32)$ & $0.11(0.05)$ & $0.00(0.00)$ & 313 & $10.13(0.49)$ & $0.05(0.00)$ \\
\hline 283 & $15.14(3.70)$ & $0.16(0.04)$ & $2.60(0.54)$ & $3.73(0.64)$ & $0.82(0.23)$ & $3.66(1.32)$ & $0.33(0.09)$ & $0.03(0.01)$ & 320 & $4.52(0.10)$ & $0.10(0.01)$ \\
\hline
\end{tabular}


Table 4

\begin{tabular}{|c|c|c|c|c|c|}
\hline & S-EBPR & V-EBPR & G-EBPR & R-EBPR & Moscow \\
\hline \multicolumn{6}{|c|}{ Eubacteria } \\
\hline 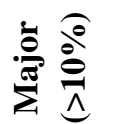 & $\begin{array}{c}\text { Proteobacteria, } \\
\text { Bacteroidetes }\end{array}$ & $\begin{array}{c}\text { Proteobacteria, } \\
\text { Bacteroidetes }\end{array}$ & $\begin{array}{c}\text { Proteobacteria, } \\
\text { Bacteroidetes }\end{array}$ & $\begin{array}{c}\text { Proteobacteria, } \\
\text { Bacteroidetes }\end{array}$ & $\begin{array}{c}\text { Proteobacteria, } \\
\text { Bacteroidetes }\end{array}$ \\
\hline 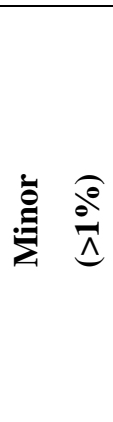 & $\begin{array}{l}\text { Planctomycetes, } \\
\text { Verrucomicrobia, } \\
\text { Firmicutes, } \\
\text { Chloroflexi }\end{array}$ & $\begin{array}{c}\text { Verrucomicrobia, } \\
\text { Actinobacteria, } \\
\text { Firmicutes, } \\
\text { Chloroflexi, } \\
\text { Planctomycetes, } \\
\text { Acidobacteria, } \\
\text { Parcubacteria }\end{array}$ & $\begin{array}{c}\text { Candidatus } \\
\text { Saccharibacteria, } \\
\text { Actinobacteria, } \\
\text { Chloroflexi, } \\
\text { Firmicutes, } \\
\text { Verrucomicrobia }\end{array}$ & $\begin{array}{c}\text { Verrucomicrobia, } \\
\text { Plactomycetes, } \\
\text { Chloroflexi, } \\
\text { Actinobacteria, } \\
\text { Acidobacteria, } \\
\text { Firmicutes }\end{array}$ & $\begin{array}{c}\text { Chloroflexi, } \\
\text { Actinobacteria, } \\
\text { Planctomycetes, } \\
\text { Firmicutes, } \\
\text { Candidatus } \\
\text { Saccharibacteria, } \\
\text { Verrucomicrobia, } \\
\text { Acidobacteria, } \\
\text { Nitrospirae }\end{array}$ \\
\hline \multicolumn{6}{|c|}{ PAOs } \\
\hline 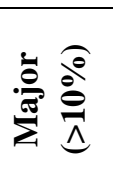 & Proteobacteria & $\begin{array}{c}\text { Proteobacteria, } \\
\text { Unknown bacterium, } \\
\text { Actinobacteria }\end{array}$ & $\begin{array}{c}\text { Proteobacteria, } \\
\text { Actinobacteria, } \\
\text { Unknown bacterium }\end{array}$ & Proteobacteria & Proteobacteria, \\
\hline 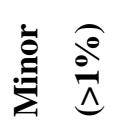 & -- & Chlamydiae & $\begin{array}{l}\text { Verrucomicrobia, } \\
\text { Chlamydiae }\end{array}$ & $\begin{array}{l}\text { Unknown bacterium, } \\
\text { Actinobacteria }\end{array}$ & $\begin{array}{l}\text { Actinobacteria, } \\
\text { Parcubacteria }\end{array}$ \\
\hline \multicolumn{6}{|c|}{ GAOs } \\
\hline 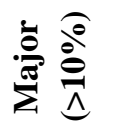 & Proteobacteria & Proteobacteria & Proteobacteria & $\begin{array}{c}\text { Proteobacteria } \\
\text { Unknown bacterium }\end{array}$ & Proteobacteria \\
\hline 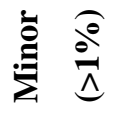 & Unknown bacterium & Unknown bacterium & Unknown bacterium & -- & Unknown bacterium \\
\hline
\end{tabular}


Table 5

\begin{tabular}{|c|c|c|c|c|c|}
\hline & S-EBPR & V-EBPR & G-EBPR & R-EBPR & Moscow \\
\hline \multicolumn{6}{|c|}{ Eubacteria } \\
\hline 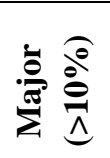 & $\begin{array}{l}\text { Rhodocyclales } \\
\text { Rhodobacterales }\end{array}$ & $\begin{array}{c}\text { unknown } \\
\text { Bacteroidetes } \\
\text { Hydrogenophilales }\end{array}$ & -- & $\begin{array}{l}\text { Hydrogenophilales } \\
\text { Sphingobacteriales }\end{array}$ & Sphingobacteriales \\
\hline 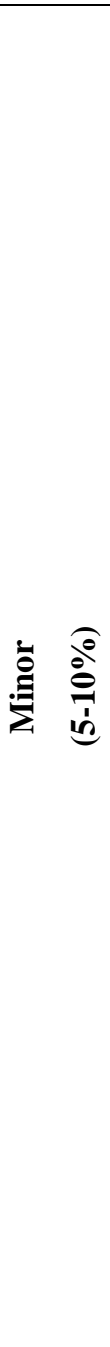 & $\begin{array}{c}\text { Flavobacteriales } \\
\text { Ohtaekwangia } \\
\text { Rhizobiales } \\
\text { Sphingobacteriales } \\
\text { Planctomycetales } \\
\text { Rhodospirillaes } \\
\text { Burkholderiales } \\
\text { Phycisphaerales } \\
\text { Xanthomonadales } \\
\text { Caulobacterales } \\
\text { Verrucomicrobiales } \\
\text { Myxococcales } \\
\text { Sphingomonadales }\end{array}$ & $\begin{array}{c}\text { Rhodocyclales } \\
\text { Sphingobacteriales } \\
\text { Burkholderiales } \\
\text { Rhizobiales } \\
\text { Cytophagales } \\
\text { Bacteriodales } \\
\text { Flavobacteriales } \\
\text { Xanthomonadales } \\
\text { Pseudomonadales } \\
\text { Rhodobacterales } \\
\text { Sphingomonadales } \\
\text { Actinomycetales } \\
\text { Rhodospirillales } \\
\text { Clostridiales } \\
\text { Caulobacterales } \\
\text { Myxococcales } \\
\text { Planctomycetales } \\
\text { Paldilineales }\end{array}$ & $\begin{array}{c}\text { Sphingobacteriales } \\
\text { Rhodocyclales } \\
\text { Saccharibacteria } \\
\text { Rhizobiales } \\
\text { Hydrogenophilales } \\
\text { Actinomycetales } \\
\text { Burkholderiales } \\
\text { Methylophilales } \\
\text { Cytophagales } \\
\text { Rhodobacterales } \\
\text { Caulobacterales } \\
\text { Flavobacteriales } \\
\text { Ohtaekwangia } \\
\text { Rhodospirillales } \\
\text { Sphingomonadales } \\
\text { Caldilineales } \\
\text { Clostridiales } \\
\text { Xerrucomicrobiales } \\
\text { Bdellovibrionales }\end{array}$ & $\begin{array}{c}\text { Burkholderiales } \\
\text { Rhizobiales } \\
\text { Rhodocyclales } \\
\text { Rhodobacterales } \\
\text { Flavobacteriales } \\
\text { Xanthomonadales } \\
\text { Ohtaekwangia } \\
\text { Planctomycetales } \\
\text { Cytophagales } \\
\text { Sphingomonadales } \\
\text { Verrucomicrobiales } \\
\text { Pseudomonadales } \\
\text { Gp4 }\end{array}$ & $\begin{array}{c}\text { Flavobacteriales } \\
\text { Burkholderiales } \\
\text { Actinomycetales } \\
\text { Rhodocyclales } \\
\text { Rhizobiales } \\
\text { Planctomycetales } \\
\text { Chloroflexales } \\
\text { Caldilineales } \\
\text { Myxococcales } \\
\text { Saccharibacteria } \\
\text { Pseudomonadales } \\
\text { Cytophagales } \\
\text { Rhodobacterales } \\
\text { Candidatus } \\
\text { Carsonella } \\
\text { Clostridiales } \\
\text { Acidimicrobiales } \\
\text { Rhodospirillales } \\
\text { Ohtaekwangia } \\
\text { Lactobacillales } \\
\text { Phycisphaerales } \\
\text { Nitrospirales }\end{array}$ \\
\hline
\end{tabular}



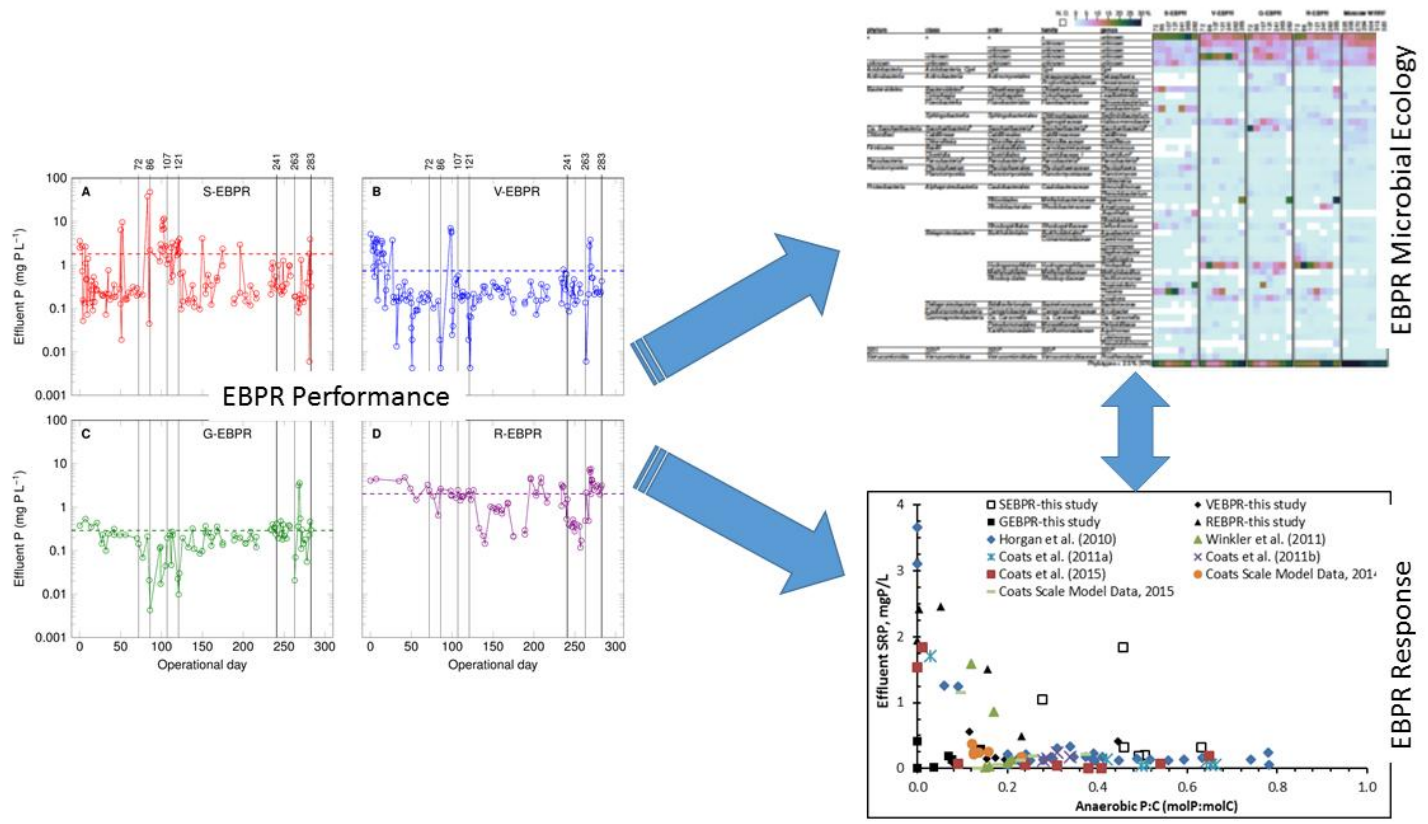